\title{
Siponimod therapy implicates Th17 cells in a preclinical model of subpial cortical injury
}

\author{
Lesley A. Ward, ${ }^{1}$ Dennis S.W. Lee, ${ }^{1}$ Anshu Sharma, ${ }^{1}$ Angela Wang, ${ }^{1}$ Ikbel Naouar, ${ }^{1}$ Xianjie I. Ma, ${ }^{1}$ \\ Natalia Pikor, ${ }^{2}$ Barbara Nuesslein-Hildesheim, ${ }^{3}$ Valeria Ramaglia, ${ }^{1}$ and Jennifer L. Gommerman ${ }^{1}$ \\ 'Department of Immunology, University of Toronto, Toronto, Ontario, Canada. Institute of Immunobiology, Kantonsspital \\ St Gallen, St Gallen, Switzerland. ${ }^{3}$ Novartis Institutes for BioMedical Research, Basel, Switzerland.
}

\begin{abstract}
Subpial demyelination is a specific hallmark of multiple sclerosis and a correlate of disease progression. Although the mechanism(s) that mediate pathogenesis in the subpial compartment remain unclear, it has been speculated that inflammation in the overlying meninges may be associated with subpial injury. Here we show that adoptive transfer of proteolipid proteinprimed Th17 cells into SJL/J recipient mice induces subpial demyelination associated with microglial/macrophage activation, disruption of the glial limitans, and evidence of an oxidative stress response. This pathology was topologically associated with foci of immune cells in the meninges and occurred in the absence of measurable anti-myelin oligodendrocyte glycoprotein IgM or IgG antibodies. To test the role of brain-infiltrating leukocytes on subpial injury, we modulated sphingosine 1-phosphate (S1P) receptor ${ }_{1,5}$ activity with BAF312 (siponimod) treatment. Administration of BAF312, even after adoptively transferred T cells had entered the brain, significantly ameliorated clinical experimental autoimmune encephalomyelitis and diminished subpial pathology, concomitant with a selective reduction in the capacity of transferred $\mathrm{T}$ cells to make Th17 cytokines. We conclude that sustained subpial cortical injury is associated with the capacity for brain-resident T cells to produce Th17 cytokines, and this pathological process occurs in an $\mathrm{S} 1 \mathrm{P}$ receptor ${ }_{1,5}$-dependent manner.
\end{abstract}

Authorship note: LAW and DSWL contributed equally to this work. VR and JLG contributed equally to this work.

Conflict of interest: JLG held a sponsored research agreement with Novartis that funded this work and currently holds funding from EMD Serono. VR received a consulting honorarium from EMD Serono and Fluidigm. BNH is an employee of Novartis.

Copyright: () 2020, American Society for Clinical Investigation.

Submitted: August 9, 2019 Accepted: November 25, 2019 Published: December 10, 2019

Reference information: JCI Insight. 2020;5(1):e132522.

https://doi.org/10.1172/jici.

insight.132522.

\section{Introduction}

Subpial cortical gray matter demyelination $(1,2)$ is a specific feature of multiple sclerosis (MS) pathology (3). From the earliest stages of disease (4), subpial lesions show evidence of both neuronal (5) and axonal (6) damage. In addition, subpial lesions are an early prognostic marker for the conversion to clinically definite MS (7) and correlate with long-term cognitive and physical impairment $(8,9)$. The absence of major blood-brain barrier disturbances (10), a paucity of parenchymal immune cell infiltration $(1,11)$, and inconsistent findings of complement deposits within subpial cortical demyelinating lesions in human autopsy tissue $(12,13)$ suggest that subpial cortical injury either proceeds independently of inflammation or involves inflammatory mechanisms that differ substantially from those underlying classic deep white matter demyelinating lesions (reviewed in ref. 14).

More recently, MS brain biopsies have identified cortical demyelinated lesions that are associated with T cell and myeloid cell infiltration (4), as well as a signature of innate and adaptive immune activation genes (3). Several reports in patients with progressive disease $(4-6,15-18)$ as well as relapsing-remitting (4) and acute MS (18) have described substantial meningeal inflammation that strongly associates with subpial cortical injury, suggesting that leukocytes in the leptomeninges may be directly or indirectly mediating this pathology. However, surprisingly little is known about the cellular and humoral mediators contributing to cortical demyelination. This is partly due to the fact that brain bank samples are, by necessity, cross-sectional and often derived from patients who have had the disease for decades (19). Unfortunately, only a limited number of animal models can mimic subpial cortical demyelination, and in these particular models, cortical demyelination is not associated with aggregates of meningeal immune cells $(20,21)$. This does not reflect the human MS scenario, where cortical pathology is clearly associated with meningeal inflammation $(4-6,15-17)$.

In this study we characterized subpial brain pathology in a mouse model of experimental autoimmune encephalomyelitis (EAE) driven by adoptively transferred Th17 lymphocytes. We previously showed that this model features the formation of tertiary lymphoid tissue (TLT) in the brain meninges (22). 
Here, we examined attributes of subpial pathology adjacent to meningeal TLT, including demyelination, microglial/macrophage accumulation, and oxidative damage, in different layers of the cortex. We also ascertained whether this pathology is associated with anti-myelin oligodendrocyte glycoprotein (antiMOG) antibodies versus the entry of specific $\mathrm{T}$ cell subsets into the inflamed brain.

\section{Results}

Meningeal inflammation in the brain of adoptively transferred SJL/J EAE mice is associated with cortical pathology. Using autopsy samples, clusters of immune cells of varying degrees of organization have been observed in the deep cerebral sulci as well as the leptomeninges of the cerebellum and spinal cord in patients with progressive MS (23-25). These structures are accompanied by cortical pathology, including demyelination, microglial activation, and neuronal degeneration $(5,6,15-17,25)$. We have previously shown that the adoptive transfer of proteolipid protein-primed (PLP-primed) Th17 cells into naive SJL/J recipients results in aggregates of immune cells (TLT) in the brain (22). However, we did not characterize and quantify the underlying subpial pathology in the cortex.

Accordingly, we induced passive EAE, which we term adoptive transfer (A/T) EAE, as described before (22) and collected brain tissue for both immunohistochemical and immunofluorescence analysis at the acute phase of the disease (see Supplemental Figure 1; supplemental material available online with this article; https://doi.org/10.1172/jci.insight.132522DS1). Using hematoxylin and eosin (HE) staining, we noted meningeal inflammation adjacent to the cortex and midbrain, the hypothalamus, the cerebellum, and the brainstem as well as in periventricular areas proximal to the third ventricle (see Supplemental Table 1, Supplemental Figure 2, and Figure 1, A-E). In addition, we noted perivascular cuffs of immune cells throughout the brain and extensively in the cerebellar white matter (data not shown).

Next we examined the pathological attributes of the brain parenchyma proximal to meningeal TLT at the acute stage of the disease. Using immunostaining for PLP to detect myelin, we found that PLP staining was significantly decreased in the midbrain parenchyma proximal to the inflamed meninges in mice that had received an adoptive transfer of Th17 cells compared with healthy mice (Figure 1, F-H), suggesting that demyelination had occurred in areas underlying meningeal TLTs.

In MS patients, one hallmark of cortical pathology is microglial activation, particularly in layer 1 proximal to areas of meningeal inflammation (5). To test this attribute in A/T SJL/J EAE, we stained for microglia/macrophages using the pan-microglial/macrophage ionized calcium-binding adaptor molecule 1 (Iba-1) marker and examined the subpial midbrain parenchyma of control and A/T SJL/J EAE mice. Compared with control mice, midbrain areas adjacent to meningeal TLT contained a significantly higher number of Iba- $1^{+}$microglia/macrophages that was not observed in control mice (Figure 1, I-K). This suggests that microglia/macrophage activation occurs in areas underlying meningeal TLTs.

In a subset of MS patients who have clusters of immune cells in the meninges, a gradient of demyelination and microglia activation from the pia mater inward has been described in areas of the cortex adjacent to the aggregates of meningeal immune cells (5). To test whether this is the case in A/T SJL/J EAE mice, we examined the density of PLP and Iba- 1 staining across layers 1 and 2/3 of the TLT-proximal and nonTLT-proximal subpial somatosensory cortex (Figure 2A). Compared with control mice, A/T SJL/J EAE mice showed a gradient of demyelination, with the most pronounced demyelination in TLT-proximal layer 1 compared with the deeper cortical layers 2 and 3 (Figure 2, B-E). In addition, A/T SJL/J EAE mice showed a gradient of microglia/macrophage activation that was most apparent in TLT-proximal layer 1 compared with layers $2 / 3$ (Figure 2, F-I). To determine whether the density of remyelinating cells (oligodendrocytes) was affected by adjacent meningeal TLT, we stained for tubulin polymerization promoting protein (Tppp/ p25), a marker of myelinating oligodendrocytes (26). Unlike the gradient of demyelination that we observed in A/T SJL/J EAE mice (Figure 2, B-E), we did not observe any differences in Tppp/p25 staining across the layers of the cortex, nor did we observe an alteration in Tppp/p25 staining in A/T SJL/J EAE mice compared to control animals (Figure 2, J-M), suggesting that myelin damage is not accompanied by the loss of the cell responsible for remyelination. Lastly, the astrocyte layer that forms the glial limitans separating the meninges from the underlying cortex was examined using glial fibrillary acidic protein (GFAP) staining. We observed that compared to control mice, A/T SJL/J mice exhibited disruption of the glial limitans and an overall increase in $\mathrm{GFAP}^{+}$reactive astrocytes in the deeper cortical layers 2/3 (Figure 2, N-Q).

In summary, SJL/J mice that receive an adoptive transfer of PLP-primed Th17 cells exhibit not only meningeal inflammation in the brain, but also cortical pathology (demyelination, microgliosis, and erosion of the glial limitans), particularly in layer 1 of the cortex, closest to meningeal TLT. 
A
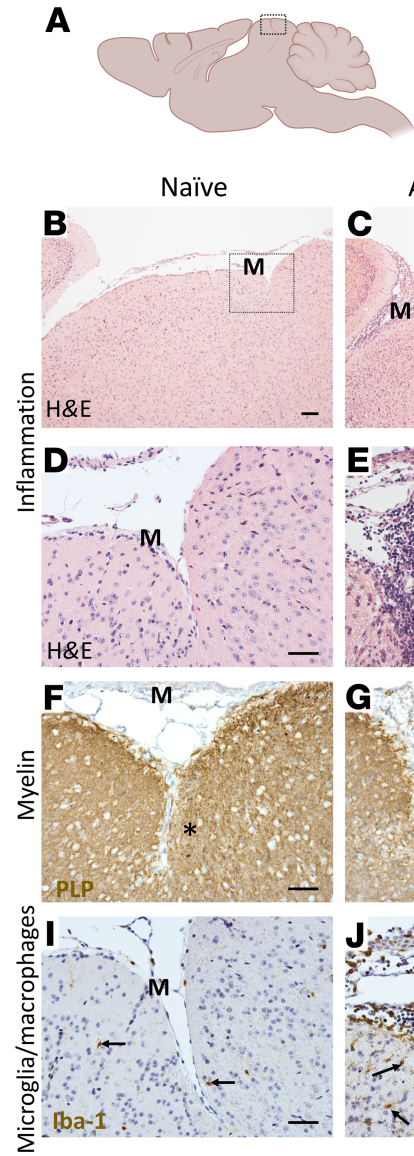

A/T SJL/J EAE
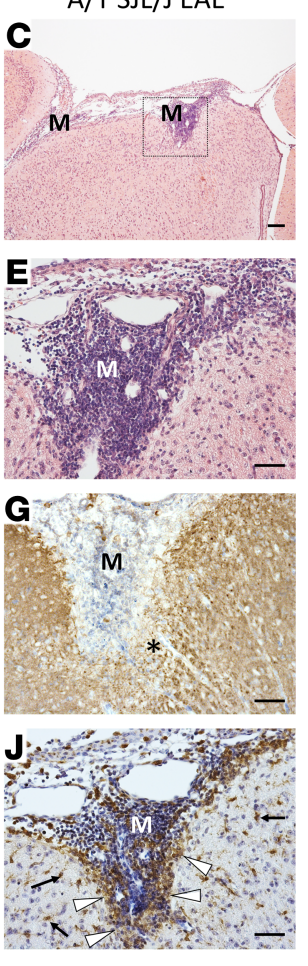
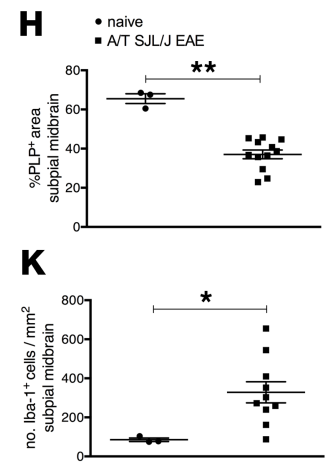

Figure 1. Meningeal inflammation in SJL/J adoptively transferred experimental autoimmune encephalomyelitis mice. (A) Diagram of the mouse brain sectioned through the sagittal neuraxis, showing areas of the midbrain imaged in $\mathbf{B}-\mathbf{C}, \mathbf{I}$, and $\mathbf{J}$. (B-E) Assessment of meningeal inflammation by hematoxylin and eosin (HE) staining in naive mice $(n=3)(\mathbf{B}$ and $\mathbf{D})$ and in adoptively transferred SJL/J experimental autoimmune encephalomyelitis (A/T SJL/J EAE) mice at day 11 (acute phase; $n=12$ ) ( $\mathbf{C}$ and $\mathbf{E}$ ). Boxes in $\mathbf{B}$ and $\mathbf{C}$ denote areas that are magnified in $\mathbf{D}$ and $\mathbf{E}$. (F and $\mathbf{G}$ ) Immunostaining for proteolipid protein (PLP), visualizing myelin, in the midbrain parenchyma adjacent to the meninges (M) in naive and A/T SJL/J EAE mice. In G, the asterisk indicates the area of subpial demyelination. (H) Quantitative analysis of the percentage of area covered by PLP staining in the subpial midbrain for each group. (I and J) Immunostaining for the ionized calcium binding adapter molecule 1 (Iba-1), a marker of microglia/macrophages in the midbrain parenchyma adjacent to the meninges in (I) naive and (J) A/T SJL/J EAE mice. In J, arrows highlight microglia/macrophages throughout the parenchyma. Arrowheads highlight aggregates of microglia/ macrophages in proximity to the pia mater. (K) Quantitative analysis of the density of $\mathrm{Iba}-1^{+}$cells in the subpial midbrain areas for each group. Values are shown as mean $\pm \mathrm{SEM}$. ${ }^{*} P<$ 0.05 , and ${ }^{* *} P<0.01$, Mann-Whitney U. Scale bars: $200 \mu \mathrm{m}$ (B and C), $50 \mu \mathrm{m}$ (I and J).

Cortical pathology in the brain of A/T SJL/J EAE mice occurs independent of anti-MOG antibodies. Cortical pathology can be induced in rodents by combining immunization with a myelin protein (MOG) with stereotaxic injection of TNF- $\alpha$ or IFN- $\gamma$. In such cases, generation of anti-MOG antibodies is either correlated with or required for subpial demyelination $(20,21,27)$. To test whether anti-MOG antibodies are associated with cortical pathology in A/T SJL/J EAE, we measured anti-MOG antibody levels in serum from these mice as well as C57BL/6 mice immunized with human recombinant MOG (hMOG) protein as a technical positive control because hMOG-immunized C57BL/6 mice generate anti-MOG IgG antibodies that are required for the manifestation of clinical disease (28). As a negative control, we examined anti-MOG antibody levels in hMOG-immunized $\mathrm{Aicda}^{-/-}$mice, which can only generate an anti-MOG IgM response and consequently show limited evidence of clinical disease $(29,30)$. As expected, anti-MOG IgG titers were detected only in hMOG-immunized C57BL/6 mice but not hMOG-immunized $\mathrm{Aicda}^{-/-}$mice. In addition, anti-MOG IgG titers were very low or not detected in naive SJL/J mice (as expected) or A/T SJL/J mice (Figure $3 \mathrm{~A}$ and Supplemental Figure 3). In terms of anti-MOG IgM responses, in agreement with our previous findings (28), immunization of C57BL/6 mice resulted in very low to undetectable titers of anti-MOG IgM presumably because MOG-specific B cells undergo rapid class switch to IgG. In contrast, hMOG-immunized $\mathrm{Aicda}^{-1-}$ mice produce anti-MOG IgM antibodies at this time point. In A/T SJL/J EAE, we found very low to undetectable levels of anti-MOG IgM compared with hMOG-immunized $\mathrm{Aicda}^{-1-}$ mice (Figure $3 \mathrm{~B}$ ). Therefore, because neither anti-MOG IgG nor anti-MOG IgM antibodies were detected at the acute phase of A/T SJL/J EAE, anti-MOG antibodies are not a requisite for the cortical pathology we observed in this model.

Next, we determined whether any cortical demyelination could be detected in hMOG-immunized mice. Examination of PLP staining revealed no evidence of cortical demyelination in the brains of C57BL/6 mice immunized with hMOG. Indeed, brains from hMOG-immunized C57BL/6 mice looked very similar in terms of myelin staining to brains from $\mathrm{hMOG}$-immunized $\mathrm{Aic}_{\mathrm{c} \mathrm{a}^{-1}}$ mice, which do not succumb to hMOG-induced EAE (28) (Figure 3, C and D). This demonstrates that the generation of anti-MOG IgG (or IgM) antibodies in response to hMOG immunization is not sufficient to induce demyelination of the cortex of C57BL/ 6 mice, although there are likely other factors lacking in C57BL/6 hMOG-immunized mice that are present in SJL/J A/T EAE mice. 
A
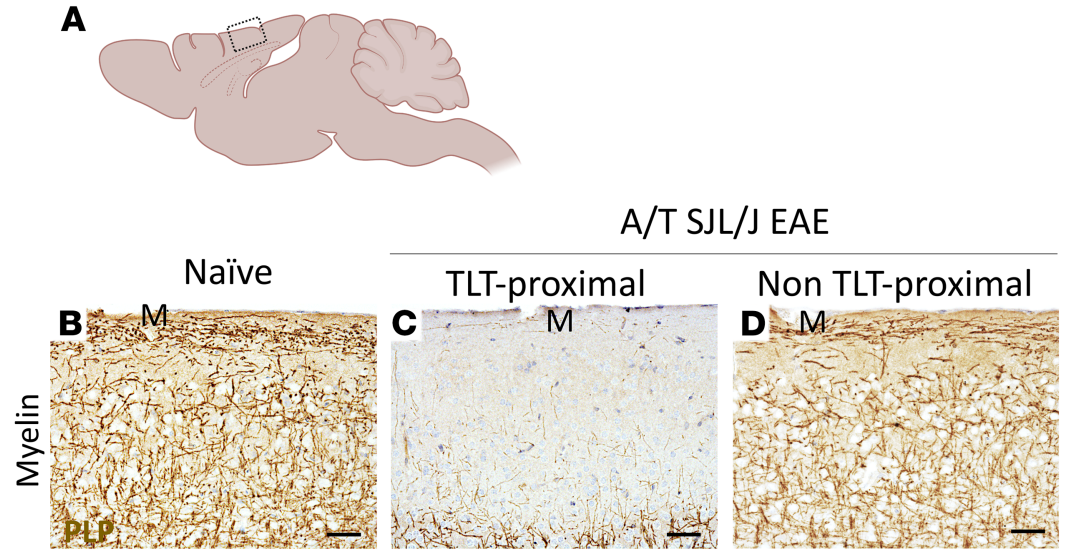

E
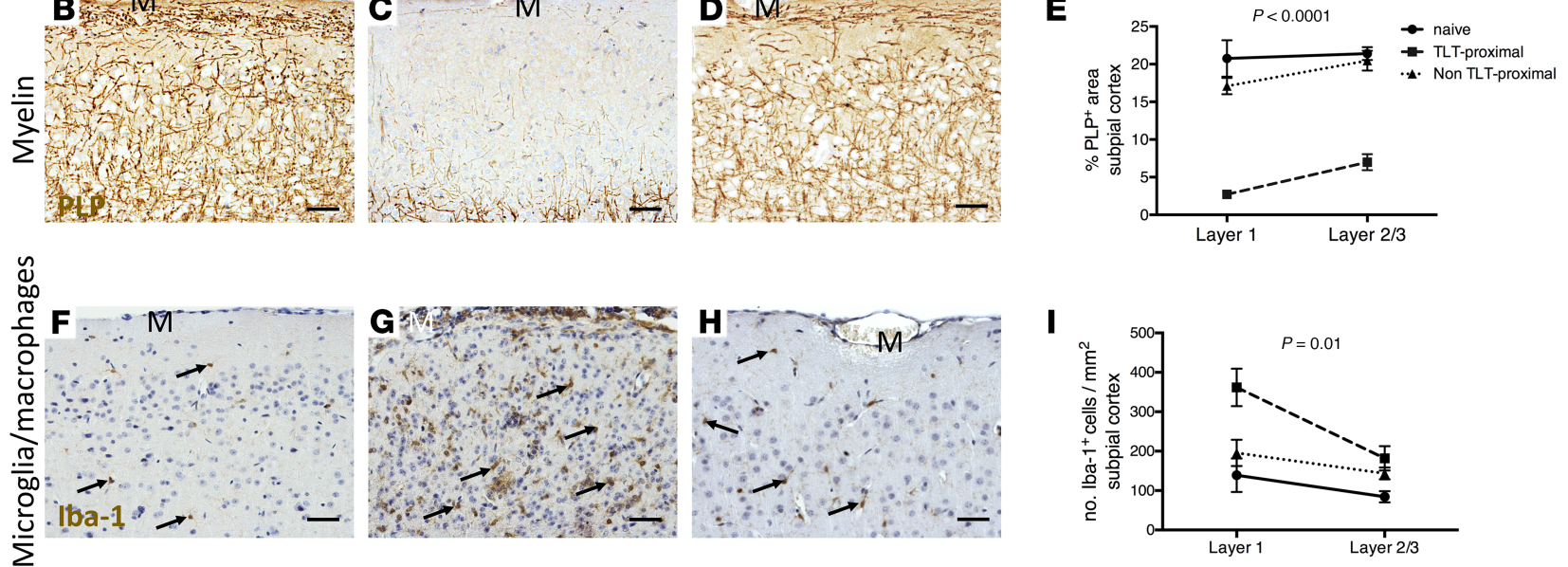

I
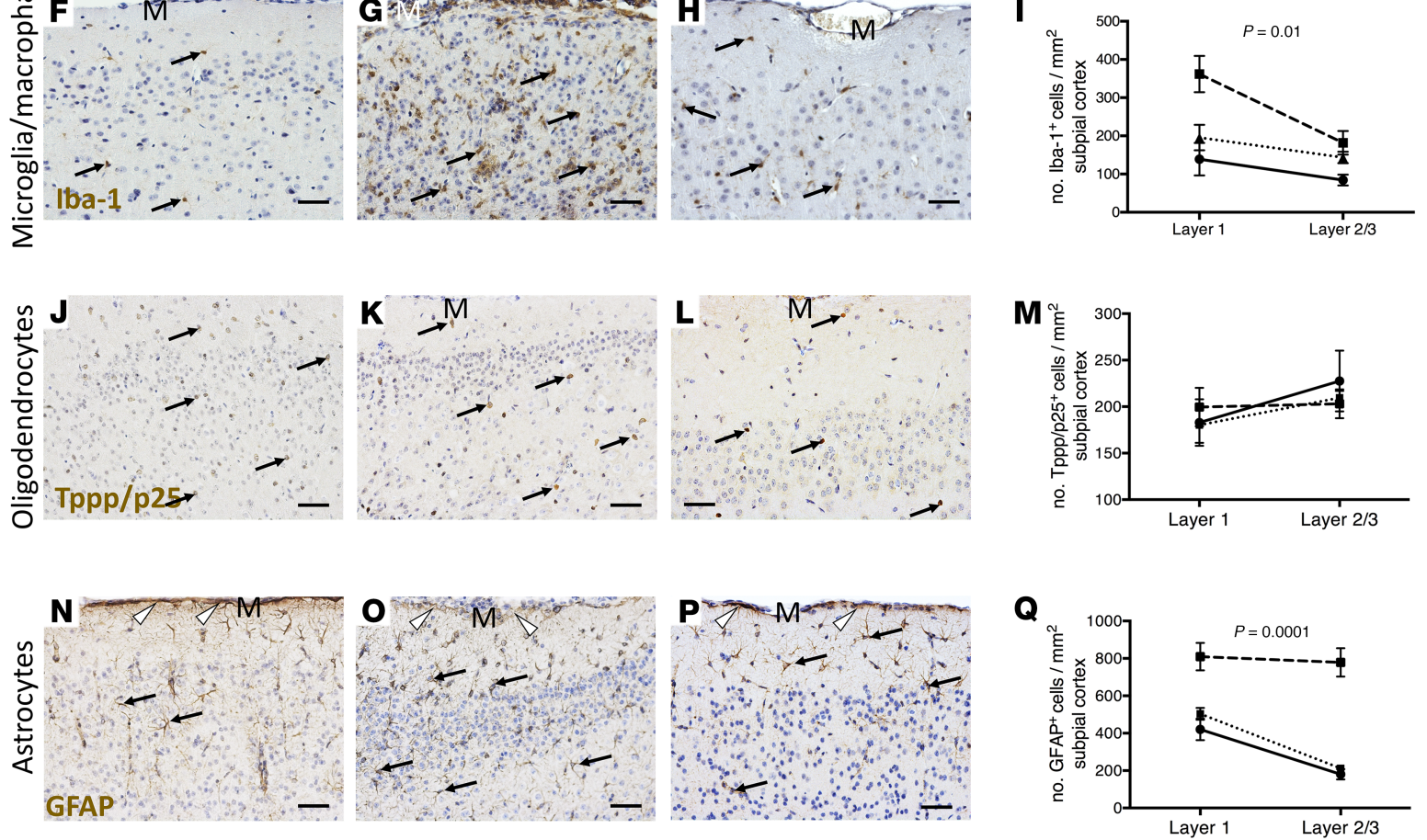

Figure 2. Meningeal inflammation is topographically related to a gradient of demyelination, reactive microglia/macrophages, and disruption of the glial limitans. (A) Diagram of the mouse brain sectioned through the sagittal neuraxis, showing areas of the somatosensory cortex imaged and analyzed in B-Q. (B-E) Immunostaining and quantitative analysis for PLP in layers 1-3 of the somatosensory cortex of naive $(n=3)$ mice as well as TLT-proximal and nonTLT-proximal layers $1-3$ of the somatosensory cortex of A/T SJL/J EAE (acute phase) mice $(n=12)$. (F-I) Immunostaining and quantitative analysis for Iba- 1 in layers 1-3 of the somatosensory cortex of naive mice and TLT-proximal and non-TLT-proximal layers 1-3 of the somatosensory cortex of A/T SJL/J EAE (acute phase) mice. Arrows depict positive staining. (J-M) Immunostaining and quantitative analysis for the tubulin polymerization promoting protein (Tppp/p25) marker of myelinating oligodendrocytes in layers 1-3 of the somatosensory cortex of naive mice and TLT-proximal and non TLT-proximal layers 1-3 of the somatosensory cortex of A/T SJL/J EAE (acute phase) mice. Arrows depict positive staining. (N-Q) Immunostaining and quantitative analysis for the glial fibrillary acidic protein (GFAP) marker of astrocytes in layers 1-3 of the somatosensory cortex of naive mice and TLT-proximal and non TLT-proximal layers 1-3 of the somatosensory cortex of A/T SJL/J EAE (acute phase) mice. Arrows depict positive staining of astrocytes and arrowheads indicate glial limitans. For all quantifications, values are shown as mean \pm SEM, and statistical significance was determined by 2-way ANOVA. M, meninges. Scale bars: $50 \mu \mathrm{m}$.

Treatment with BAF312 reduces the severity of A/T SJL/JEAE concomitant with a reduction in the frequency of donor $C D 4^{+} T$ cells producing Th17 cytokines in the brain. In A/T SJL/J EAE, adoptively transferred and endogenous $\mathrm{T}$ cells both accumulate in the brain meninges during clinically apparent disease (22). However, it is not clear which of these pools of $\mathrm{T}$ cells are responsible for the observed underlying cortical pathology. To determine the impact of sphingosine 1-phosphate (S1P) receptors on meningeal TLT and subpial pathology in SJL/J recipient mice, we administered a selective $\mathrm{S}_{1,5}$ modulator (BAF312) following adoptive 


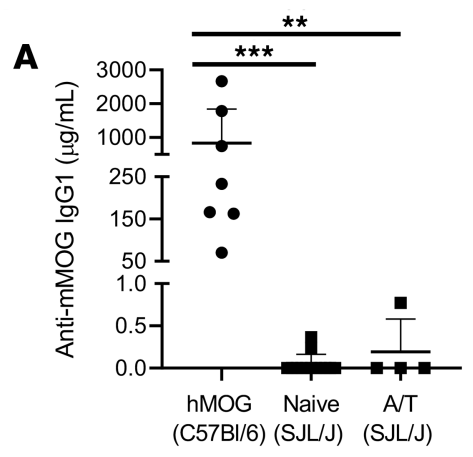

Naive

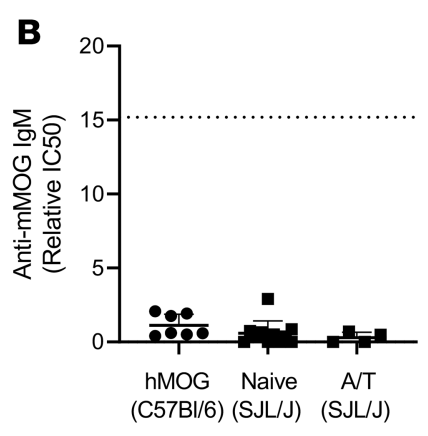

C

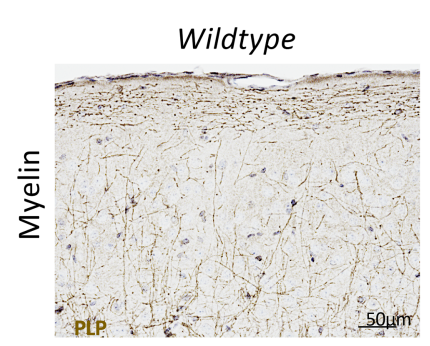

EAE (hMOG)

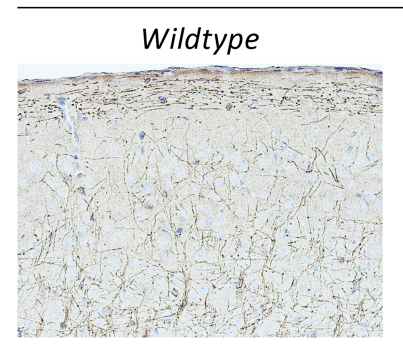

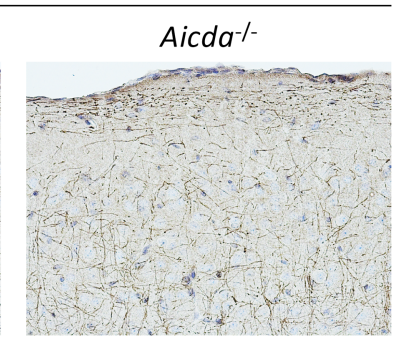

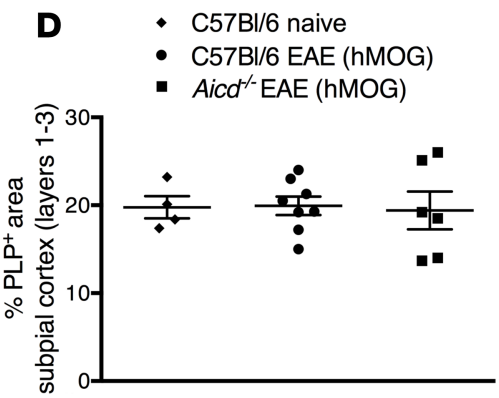

Figure 3. Alternative EAE models, with anti-MOC antibody production, do not produce significant cortical pathology. ELISA was used to measure anti-mouse MOC IgC (A) and anti-mouse MOC IgM (B) in the blood. EAE was induced in C57BL/6 mice by immunization with hMOC and by adoptive transfer in SJL/J mice. (B) Anti-MOG IgM was quantified by comparing the $\mathrm{IC}_{50}$ of dilution curves (see Methods and Supplemental Figure 3 ). Sera from Aicda $^{-/}$mice immunized with hMOG were pooled to create a "standard" lot that was run as an internal control and is denoted by the dotted line. (C) Immunostaining and (D) quantitative analysis for PLP in layers 1-3 of the somatosensory cortex of naive wild-type $(C 57 \mathrm{BL} / 6)$ mice $(n=4)$ and C57BL/6 $(n=8)$ or Aicda ${ }^{--}$mice $(n=6)$ immunized with hMOG. Values are shown as mean $\pm(\mathbf{A}$ and B) SD or (D) SEM. Statistical significance for hMOG ELISAs was determined by Mann-Whitney $U$; ${ }^{* *} P<0.01$, and ${ }^{* * *} P<0.0001$. Statistical analysis for histology was done by Kruskal-Wallis with Dunn's correction for multiple comparisons. Sera were collected from A/T SJL/J EAE mice on day 11 after adoptive transfer, and sera from hMOC mice were collected during the chronic phase of EAE. Scale bars: $50 \mu \mathrm{m}$.

transfer of T cells stimulated ex vivo under Th17-skewing conditions. Because transferred proinflammatory $\mathrm{T}$ cells appear in the brain starting at day 5 after transfer (22), we administered BAF312 to recipient SJL/J mice at days 3, 5, or 8 after adoptive transfer and assessed EAE severity using a 16-point composite clinical scoring index, comparing the 3 treatment groups with a group of vehicle control-treated recipient mice (Figure 4A). Treatment beginning at day 3 resulted in a complete inhibition of EAE symptoms, whereas treatment beginning at day 5 resulted in a significant but incomplete reduction in disease symptoms. In contrast, treatment beginning at day 8 did not alter clinical disease (Figure 4, B and C). Similar results were observed when we recorded weight loss as an additional endpoint (Figure 4D). Based on these findings, we conclude that the activity of $\mathrm{S}_{1,5}$ is required for the induction of EAE by preprimed Th17 cells.

To ascertain the role of $\mathrm{S}_{1} \mathrm{P}_{1,5}$ signaling on different subsets of $\mathrm{CD}^{+} \mathrm{T}$ cells, we tracked the donor-derived $\left(\mathrm{CD} 45.2^{+}\right)$transferred $\mathrm{CD} 4^{+} \mathrm{T}$ cells versus endogenous $\left(\mathrm{CD} 45.1^{+}\right) \mathrm{CD}^{+} \mathrm{T}$ cells in BAF312- versus vehicle-treated A/T SJL/J EAE mice (see gating strategy in Supplemental Figure 4). Although clinical disease was reduced in mice treated with BAF312 beginning at day 5 , we could nevertheless detect $\mathrm{CD}^{+}{ }^{+} \mathrm{T}$ cells in the brain of $\mathrm{A} / \mathrm{T} \mathrm{SJL} / \mathrm{J}$ EAE mice when we analyzed the entire tissue by flow cytometry. BAF312 treatment resulted in a significant reduction in endogenous $\left(\mathrm{CD} 45.1^{+} \mathrm{CD} 4^{+}\right)$, but not transferred $\left(\mathrm{CD} 45.2^{+} \mathrm{CD} 4^{+}\right)$, $\mathrm{T}$ cells, when expressed as a frequency of total live cells in the brain (Figure 5A). In terms of absolute numbers, there was also a nonsignificant trend toward a reduction in endogenous $\mathrm{T}$ cells (Figure 5B).

Next, we used intracellular flow cytometry to determine the capacity of both endogenous and transferred $\mathrm{CD}^{+} \mathrm{T}$ cells to produce IFN- $\gamma$, IL-17, or GM-CSF. BAF312 treatment resulted in a reduction in $\mathrm{IL}_{-1} 7^{+}$and $\mathrm{GM}-\mathrm{CSF}^{+}$transferred $\mathrm{T}$ cells when expressed as a frequency of total transferred $\mathrm{T}$ cells (Figure 5C). On the other hand, we did not observe a BAF312-driven reduction in $\mathrm{IL}_{-1} 7^{+}$and $\mathrm{GM}-\mathrm{CSF}^{+}$ endogenous $\mathrm{T}$ cells when expressed as a frequency of total endogenous $\mathrm{T}$ cells but instead noted an 
A

Treatment BAF312 (3mg/kg p.o.) or CMC ( Vol/Vol) $\downarrow \downarrow \downarrow \downarrow \downarrow \downarrow \downarrow \downarrow$

$\downarrow \downarrow \downarrow \downarrow \downarrow \downarrow$

$\downarrow \downarrow \downarrow$

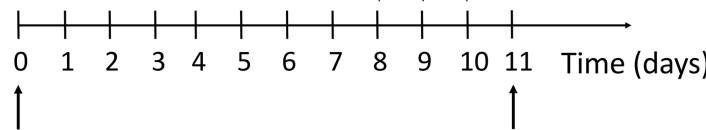

A/T of CD4-selected Th17 cells from PLP ${ }_{139-151}$-immunized SJL/J donors ( $20 \mathrm{mln}$ cells i.p.)

B
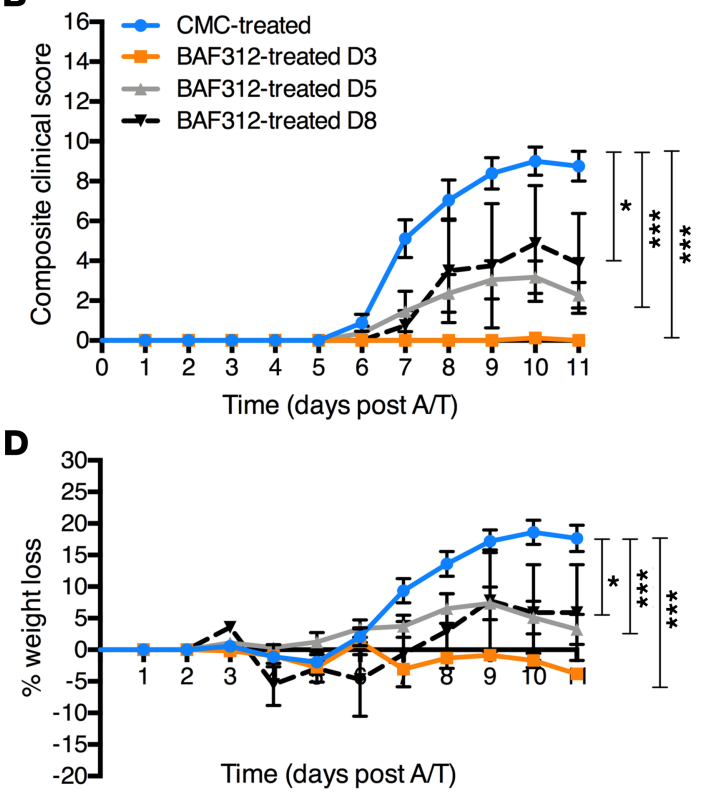

End-point

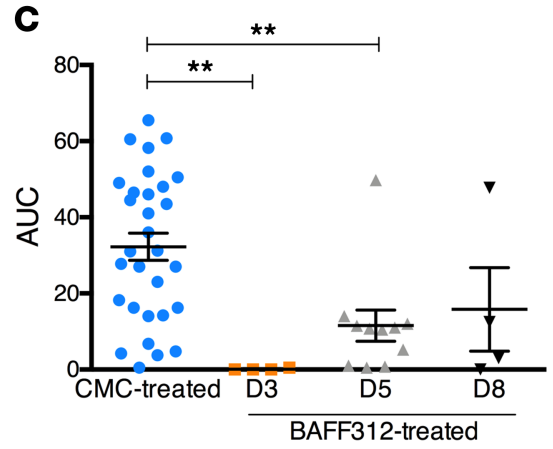

Figure 4. Therapeutic treatment with BAF312 ameliorates clinical outcome in A/T SJL/J EAE mice. (A) Schematic of the A/T SJL/J EAE protocol integrating the BAF312 or vehicle control (carboxymethylcellulose, CMC) treatment timeline. (B) Composite clinical score (16-point scale) of A/T SJL/J EAE recipient mice treated with CMC $(n=30)$ or BAF312 over time (from day $3, n=4$; from day $5, n=12$; from day $8, n=4$ ). (C) Area under the curve (AUC) cumulative disease score for each group. (D) Percentage weight loss of A/T SJL/J EAE recipient mice treated with BAF312 or vehicle control over time. Values are shown as mean $\pm \mathrm{SEM}$, and statistical significance was determined by 2 -way ANOVA, with ${ }^{*} P<0.05$ and ${ }^{* *} P<0.001$ in $\mathbf{B}$, or by 1 -way ANOVA, with ${ }^{* *} P<0.01$ in $\mathbf{C}$.

increase in frequency of endogenous $\mathrm{T}$ cells producing IFN- $\gamma$ (Figure 5E). Thus, when we examined the capacity of $\mathrm{CD} 4^{+} \mathrm{T}$ cells to produce cytokines, there appeared to be a reduction at the level of Th17 cytokines (IL-17, GM-CSF) in the transferred T cell compartment but not in the endogenous T cell compartment in BAF312-treated mice.

To further corroborate these findings, we also examined the capacity of both transferred and endogenous $\mathrm{T}$ cells to make more than one cytokine. Interestingly, we observed a reduction in IFN- $\gamma^{+} \mathrm{IL}-17^{+}$ and $\mathrm{GM}-\mathrm{CSF}^{+} \mathrm{IL}-17^{+}$transferred $\mathrm{T}$ cells when expressed as a frequency of total transferred $\mathrm{T}$ cells in BAF312-treated mice (Figure 5C). This was not observed in the endogenous $\mathrm{T}$ cell compartment in BAF312-treated mice (Figure 5E). These changes in the frequency of cytokine-producing cells were less obvious when we examined absolute numbers of brain-resident cells (Figure 5, D and F).

Collectively, these data show that compared with treatment with vehicle control, treatment with BAF312 results in a reduction of endogenous $\mathrm{T}$ cells, when expressed as a frequency of total live cells in the brain, as well as a selective reduction in the capacity for disease-initiating transferred $\mathrm{T}$ cells to produce Th17 cytokines.

Treatment with BAF312 impairs the formation of meningeal TLT in A/T SJL/J EAE mice. To determine whether the activity of $\mathrm{S}_{1} \mathrm{P}_{1,5}$ is required for remodeling of the subarachnoid space of the EAE brain, we examined whether meningeal TLTs form in BAF312-treated A/T SJL/J EAE mice. Like the flow cytometry experiments, in these experiments we focused on the day 5 treatment modality because A/T T cells have reached the brain of recipient mice by this time point (22), and treatment beginning on day 5 resulted in a significant reduction, but not an elimination, of clinical symptoms (Figure 4, B and C). As before (22), 
- CMC-treated

BAF312-treated D5
A
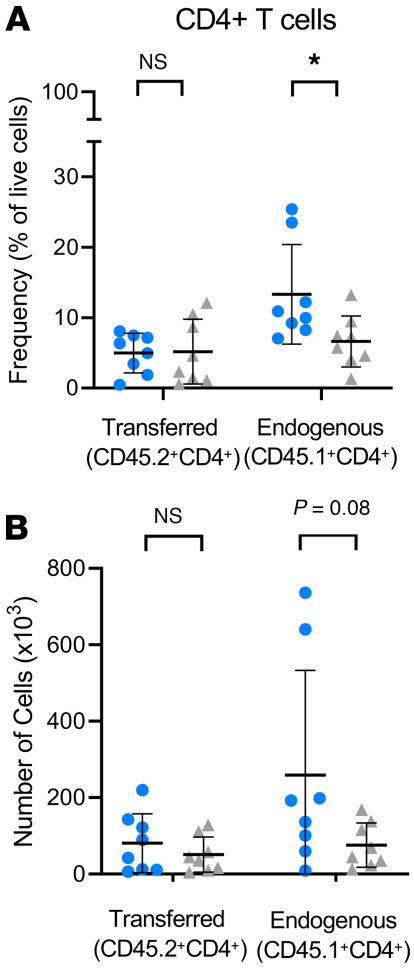

C

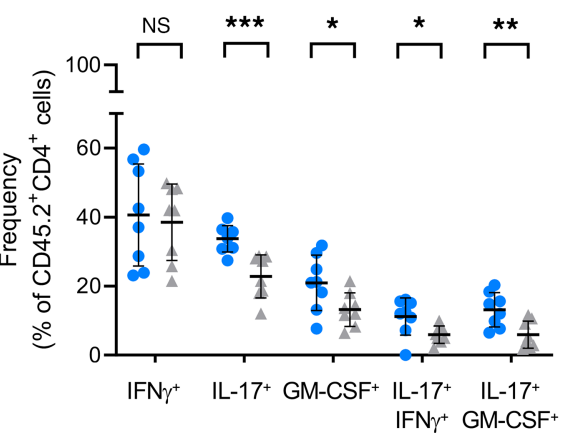

D

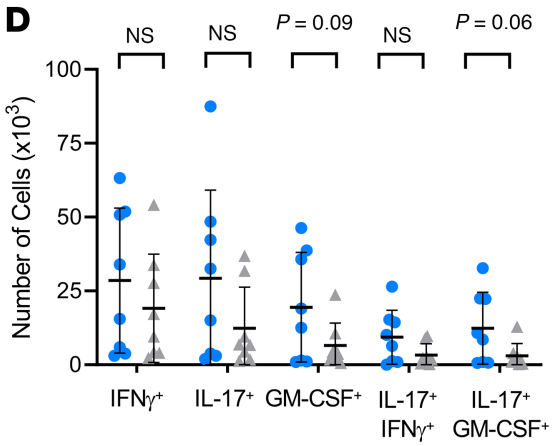

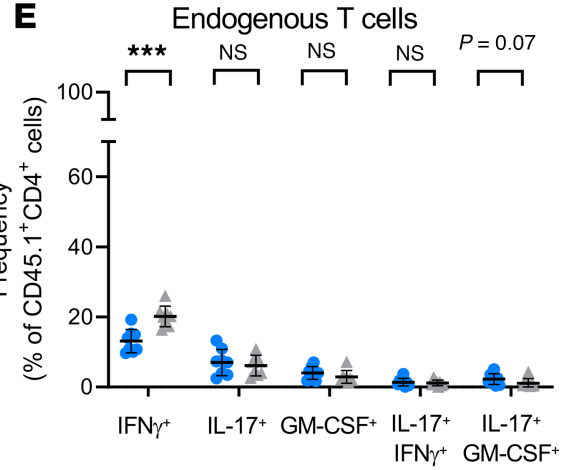

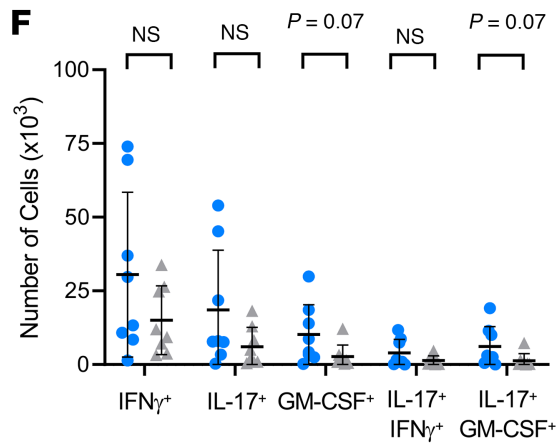

Figure 5. BAF312 treatment reduces proinflammatory cytokine production from A/T CD4+ $\mathbf{T}$ cells in the brain. (A-F) Single-cell suspensions from the brains of BAF312- versus control-treated mice at day 11 after adoptive transfer were stained for CD4 as well as CD45.1 versus CD45.2 alleles identifying endogenous (CD45.1 host-derived) versus transferred (CD45.2 donor-derived) lymphocytes. (A and B) Total CD4 ${ }^{+}$T cells were assessed, and (C-F) cytokine production from CD4+ T cells was measured using intracellular staining for IFN- $\gamma$, IL-17, and GM-CSF as well as for both IFN- $\gamma$ and IL-17 or both GM-CSF and IL-17. Values are expressed either as a frequency of total live cells (A, C, E) or as an absolute number of brain-resident cells (B, D, F). Two experiments were combined for analysis; $n=8-9$ mice per group. Values are shown as mean $\pm \mathrm{SD}$, and statistical significance was determined by Mann-Whitney $U$, with ${ }^{*} P<0.05,{ }^{*} P<0.01$, and ${ }^{* *} P<0.001$.

we found that transfer of Th17 cells resulted in accumulation of immune cells in the meninges (Figure $6, \mathrm{~A}-\mathrm{C}$ ) with an expansion of the fibronectin network within the meningeal subarachnoid space (Figure 6, D-G) and an accumulation of leukocytes within these areas (Figure 6, H-R). Interestingly, although BAF312-treated mice exhibited some clinical disease (Figure 4, B and C), fibronectin network formation in the meninges was reduced by $47 \%$ compared with control-treated mice (Figure 6, D-G). Moreover, the residual extracellular matrix elaboration was not sufficient to support the accumulation of $\mathrm{B} 220^{+}$or $\mathrm{CD}^{+}$ cells because these were significantly decreased by $85 \%$ and $70 \%$, respectively, in A/T SJL/J EAE mice treated with BAF312 compared with vehicle control (Figure $6, \mathrm{H}-\mathrm{R}$ ). Therefore, $\mathrm{S}_{1} \mathrm{P}_{1,5}$ signaling is required to optimize the formation of meningeal TLT in the subarachnoid space of A/T SJL/J EAE mice.

Treatment with BAF312 prevents subpial pathology in A/T SJL/J EAE mice. To determine whether subpial pathology is affected by BAF312 treatment, we quantified the content of myelin, microglia/macrophages, and myelinating oligodendrocytes in subpial regions of the somatosensory cortex, as well as the integrity of the glial limitans by staining for astrocytes. Compared with administration of vehicle control, administration of BAF312 beginning at day 5 after adoptive transfer resulted in a sparing of myelin along with a corresponding reduction in Iba- $1^{+}$microglia/macrophage and in $\mathrm{GFAP}^{+}$astrocyte numbers in the subpial cortex. Myelinating oligodendrocytes, whose numbers were not changed upon adoptive transfer of Th17 cells, were also not changed in response to BAF312 treatment (Figure 7).

Next, we examined the impact of adoptive transfer of Th17 cells on neuronal damage. Using immunostaining for the neuronal nuclei (NeuN) marker of neurons, we first quantified neuronal density in layers $1-3$ of the somatosensory cortex. We found that adoptive transfer of Th17 cells did not result in a visible 
Naïve
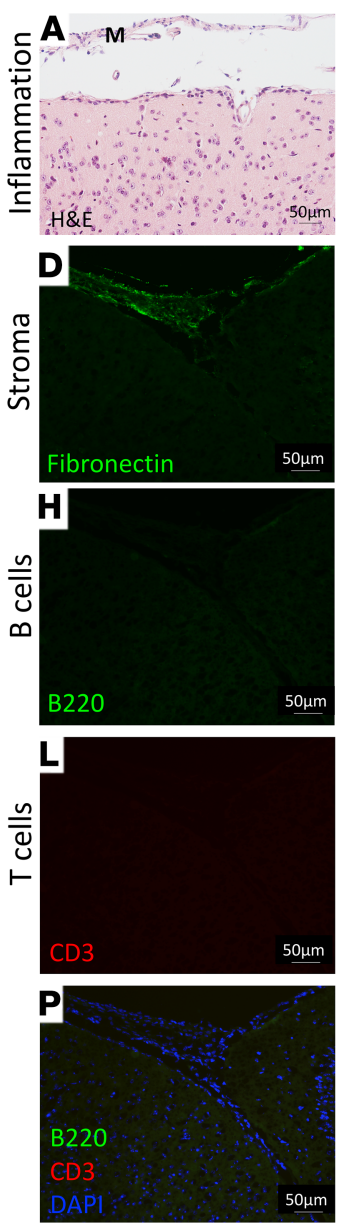

A/T SJL/J EAE
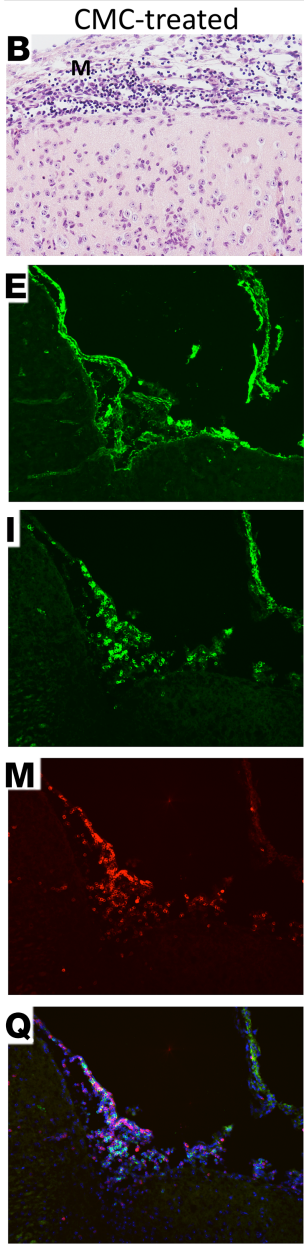
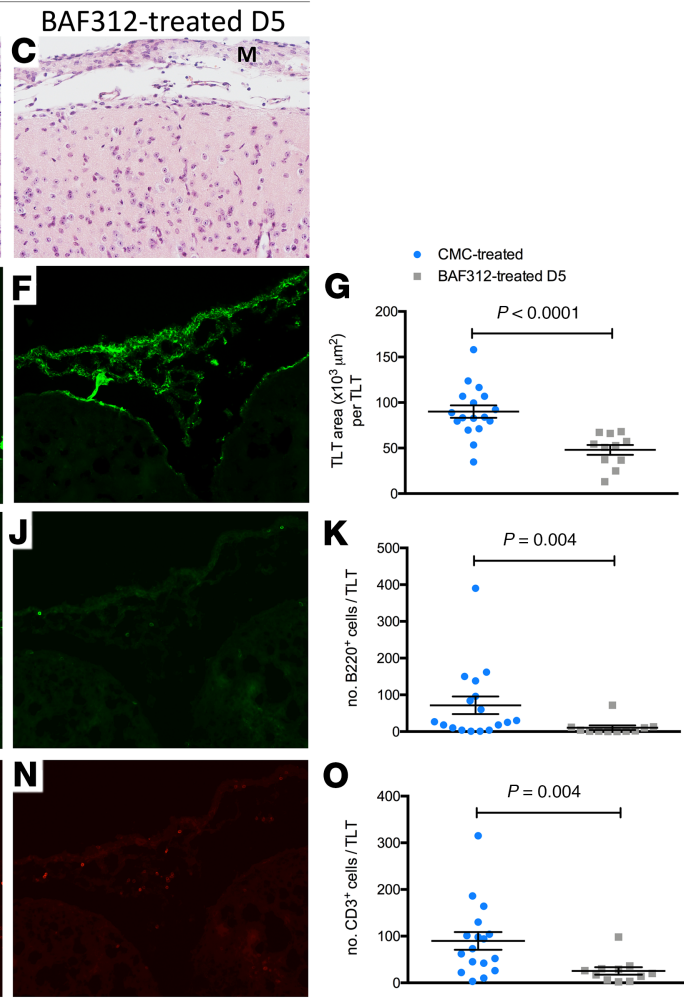

Figure 6. Therapeutic treatment with BAF312 reduces TLT area and the number of $B$ and $T$ cells in the meninges of A/T SJL/J EAE mice. (A-C) HE staining of brain sections from naive mice and A/T SJL/J EAE mice at day 11 (acute phase) treated with either vehicle control (CMC) or BAF312. (D-G) Immunofluorescent staining and quantitative analysis for the extracellular matrix protein fibronectin in brain sections from naive mice and $A / T$ SJL/J EAE mice at day 11 (acute phase) treated with either vehicle control (CMC) or BAF312. (H-K) Immunofluorescence staining and quantitative analysis for $\mathrm{B} 22 \mathrm{O}^{+} \mathrm{B}$ cells in brain sections from naive mice and A/T SJL/J EAE mice at day 11 (acute phase) treated with either vehicle control (CMC) or BAF312. (L-O) Immunofluorescence staining and quantitative analysis for CD3 ${ }^{+} \mathrm{T}$ cells in brain sections from naive mice and A/T SJL/J EAE mice at day 11 (acute phase) treated with either vehicle control (CMC) or BAF312. (P-R) Merged images of $\mathbf{H}-\mathbf{J}$ and $\mathbf{L}-\mathbf{N}$. M, meninges. For all quantifications, values are shown as mean \pm SEM, and statistical analysis was done by Mann-Whitney $U$ test. Scale bars: $50 \mu \mathrm{m}$.

loss in NeuN $\mathrm{N}^{+}$cells (Figure 8, A-C), and quantification of $\mathrm{NeuN}^{+}$cells showed that no changes in neuronal numbers occurred during A/T SJL/J EAE with or without BAF312 treatment (Figure 8D). This suggests that accumulation of meningeal immune cells during the acute stage of $\mathrm{A} / \mathrm{T} \mathrm{SJL} / \mathrm{J}$ EAE does not affect viability of neuronal cells in the cortex.

Recently, it has been shown that metabolic and oxidative injury may drive tissue pathology in the subpial cortex $(3,31,32)$, but this is only partially reflected in current experimental disease models (33). Accordingly, we measured mitochondrial heat shock protein 70 (mtHsp70), a marker of metabolic stress in mitochondria, and 8-hydroxy-2'-guanosine ( $8 \mathrm{OHG}$ ), a marker of oxidized nucleosides in RNA (and reported to detect the 8-hydroxy-2'-deoxyguanosine [8OHdG] in DNA), in neurons of layers 2 and 3 of the somatosensory cortex. We found that A/T SJL/J EAE mice show evidence of metabolic stress in mitochondria located in the subpial area of the cortex, from layers 2 through layer 3 (most obvious in pyramidal neurons) (Figure 8, $\mathrm{E}-\mathrm{G})$. Moreover, treatment with BAF312 resulted in a nonsignificant reduction (1.8-fold) in the percentage of $\mathrm{mtHSP} 70^{+}$area, whether the mice were treated from day 3 or day 5 after adoptive transfer (Figure $8 \mathrm{H}$ ). The percentages of mtHSP $70^{+}$area in the cortex of mice treated with BAF312 from day 3 or from day 5 


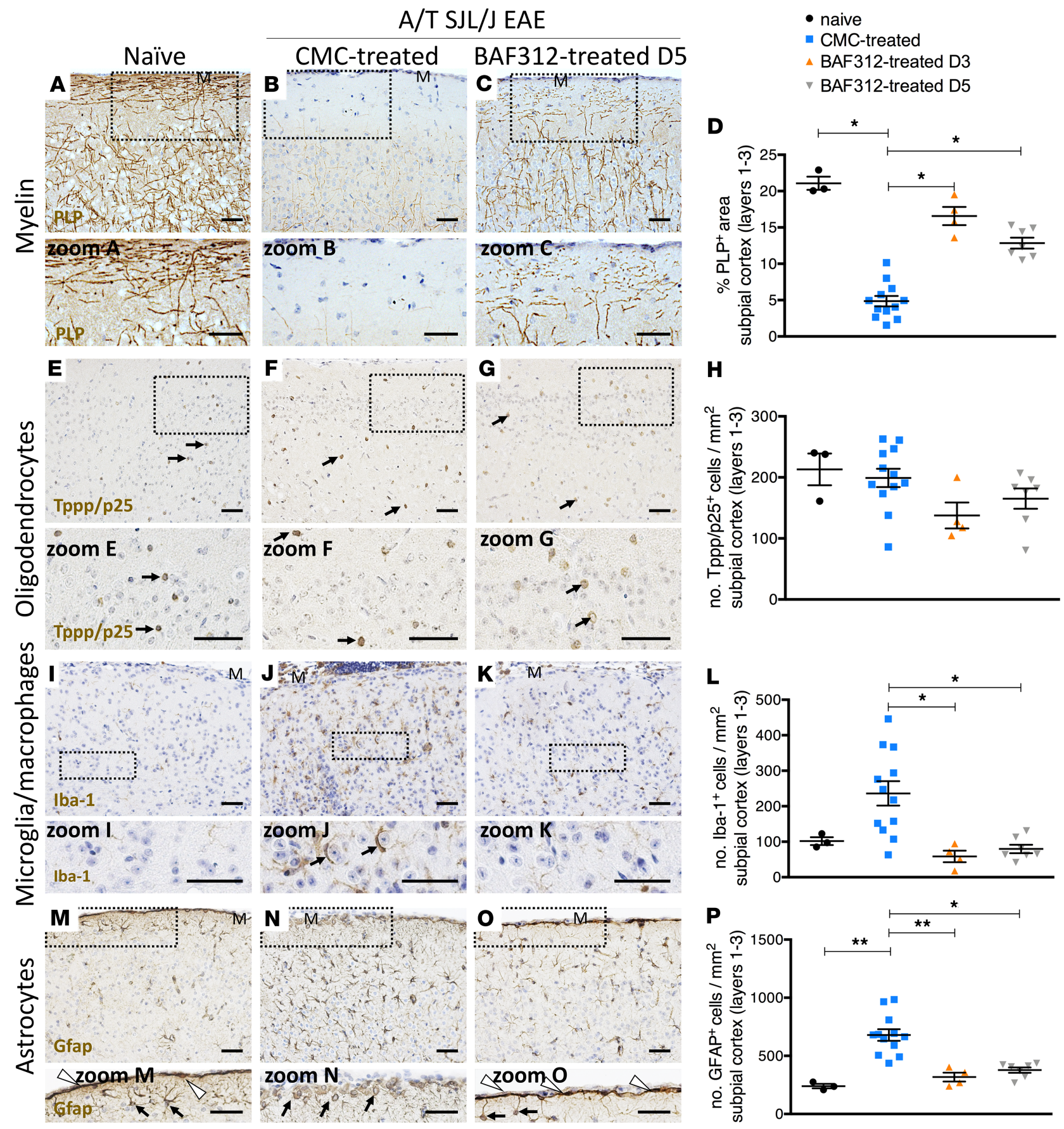

Figure 7. Therapeutic treatment with BAF312 reduces demyelination, microglia/macrophage reactivity, and glial limitans disruption in A/T SJL/J EAE mice. (A-D) Immunostaining and quantitative analysis for PLP in brain sections (layers 1-3 of the somatosensory cortex) from naive mice ( $n=3$ ) and A/T SJL/J EAE mice at day 11 (acute phase) treated with either vehicle control (CMC, $n=12$ ) or BAF312 over time (from day $3, n=4 ;$ from day $5, n$ = 7). Zoomed areas show myelin changes in layer 1 of the somatosensory cortex. (E-H) Immunostaining and quantitative analysis for the Tppp/p25 marker of myelinating oligodendrocytes in brain sections from naive mice and A/T SJL/J EAE mice treated with either vehicle control or BAF312 over time, as above. Zoomed areas show Tppp/p25+ cells (arrows) in layer 2 of the somatosensory cortex. (I-L) Immunostaining and quantitative analysis for Iba1 in brain sections from naive mice and A/T SJL/J EAE mice treated with either vehicle control or BAF312 over time, as above. Zoomed areas show microglia/macrophages closely apposed to neurons in CMC-treated A/T SJL/J EAE mice (zoom J) but not in naive (zoom I) or BAF312-treated A/T SJL/J EAE mice (zoom K). (M-P) Immunostaining and quantitative analysis for GFAP in brain sections from naive mice and A/T SJL/J EAE mice treated with either vehicle control or BAF312 over time, as above. Zoomed areas show a disrupted glial limitans and enlarged GFAP ${ }^{+}$astrocytes underlying meningeal TLT in A/T SJL/J EAE mice treated with CMC (arrows in zoom $\mathbf{N}$ ), whereas GFAP' astrocytes with processes that extend toward the pia mater and form an intact glial limitans were seen in naive (zoom M) and BAF312-treated mice (zoom $\mathbf{0}$ ). For all quantifications, values are shown as mean \pm SEM, and statistical analysis was done by Kruskal-Wallis with Dunn's correction for multiple comparisons, with ${ }^{*} P<0.05$, and ${ }^{*} P<0.01$. $\mathrm{M}$, meninges. Scale bars: $50 \mu \mathrm{m}$. 

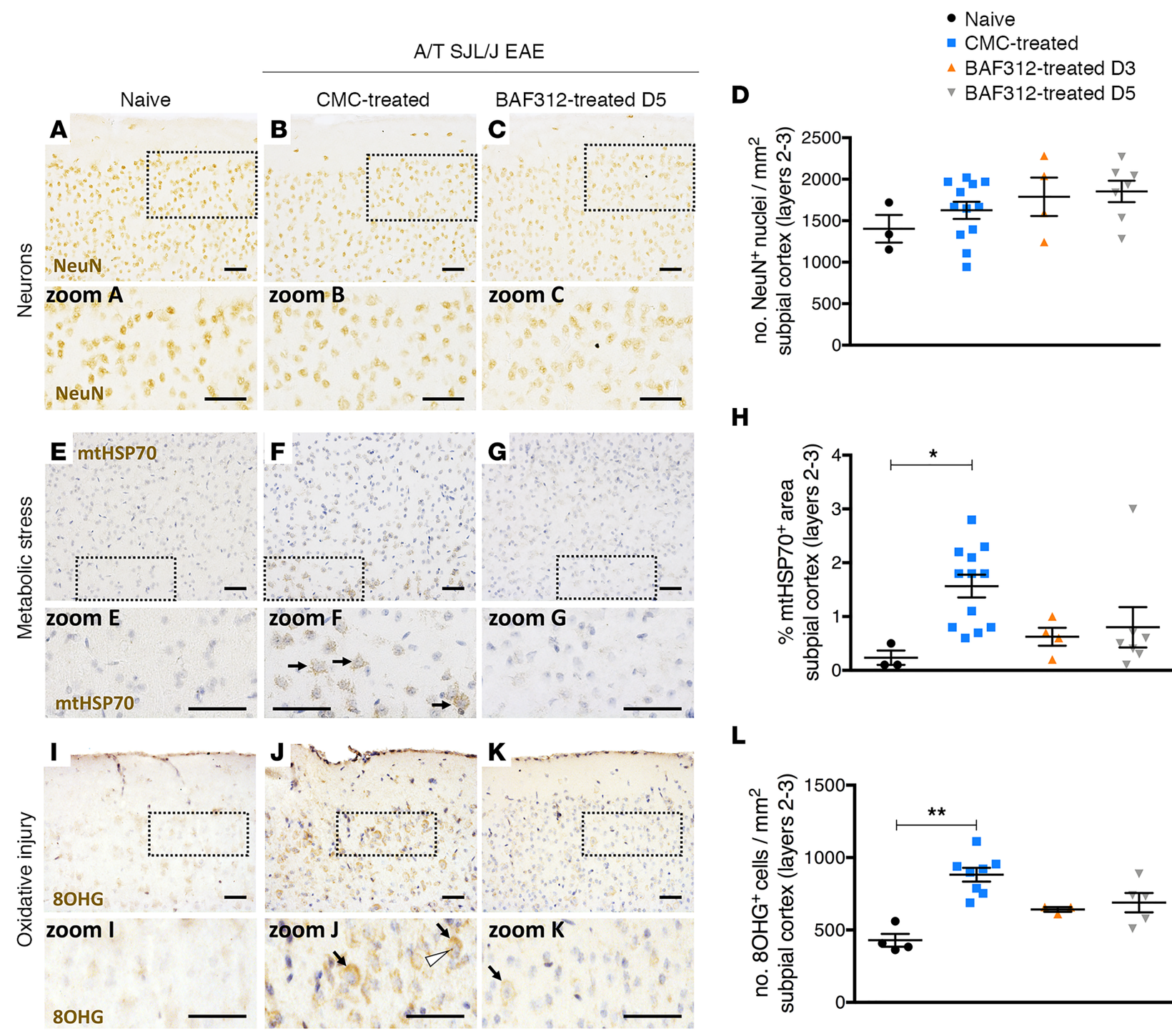

H

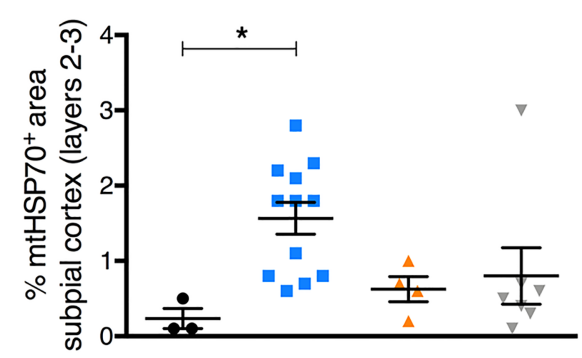

$\mathbf{L}$

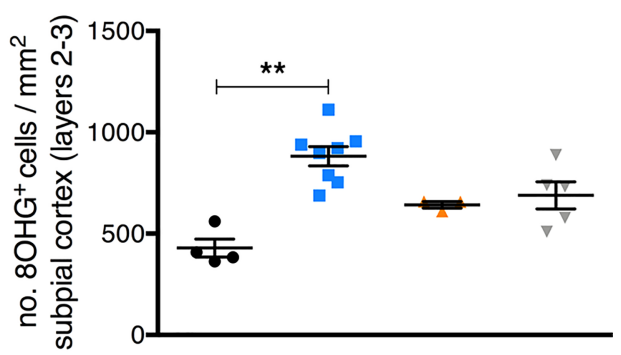

Figure 8. Therapeutic treatment with BAF312 does not affect neuronal count but reduces oxidative stress in A/T SJL/J EAE mice. (A-D) Immunostaining and quantitative analysis for the neuronal nuclei (NeuN) marker of neurons in brain sections (layers 2-3 of the somatosensory cortex) from naive mice ( $n=$ 3) and A/T SJL/J EAE mice at day 11 (acute phase) treated with either vehicle control (CMC, $n=12$ ) or BAF312 over time (from day $3, n=4 ;$ from day $5, n=7$ ). Zoomed areas show NeuN ${ }^{+}$cells in layer 2 of the somatosensory cortex. (E-H) Immunostaining and quantitative analysis for the mitochondrial heat shock protein 70 (mtHsp70) marker of metabolic stress in brain sections from naive mice and A/T SJL/J EAE mice treated with either vehicle control or BAF312 over time, as above. Zoomed areas show mtHsp70 staining of mitochondria in layer 3 neurons of the somatosensory cortex of CMC-treated A/T SJL/J EAE mice (arrows in zoom F) but not in naive (zoom E) or BAF312-treated A/T SJL/J EAE mice (zoom G). (I-L) Immunostaining and quantitative analysis for the 8-hydroxy-2'-guanosine (8OHG) marker of oxidative injury in brain sections (layers $2-3$ of the somatosensory cortex) from naive mice $(n=4)$ and A/T SJL/J EAE mice at day 11 (acute phase) treated with either vehicle control (CMC, $n=8$ ) or BAF312 over time (from day 3, $n=3 ;$ from day $5, n=5$ ). Zoomed areas show $80 \mathrm{HC}$ staining of cells in the cytoplasm (arrows) and nucleolus (arrowhead) of layer 2 neurons in the somatosensory cortex of CMC-treated A/T SJL/J EAE mice (zoom J) compared to naive (zoom I) or BAF312-treated A/T SJL/J EAE mice (zoom K). For all quantifications, values are shown as mean \pm SEM, and statistical analysis was done by Kruskal-Wallis with Dunn's correction for multiple comparisons, with ${ }^{*} P<0.05$ and ${ }^{*} P<0.01$. Scale bars: $50 \mu \mathrm{m}$.

were not significantly different from each other $(P=0.6$ by Mann-Whitney $U$ ). If these data points are combined, the BAF312-treated mice showed a significant reduction in the percentage of mtHSP70 $0^{+}$area $(1.6 \pm$ 0.7 , mean $\pm \mathrm{SD})$ compared with the control-treated mice $(0.7 \pm 0.8$, mean $\pm \mathrm{SD} ; P<0.05$ by Kruskal-Wallis with Dunn's correction for multiple comparisons). In terms of $8 \mathrm{OHG}$, we found that A/T SJL/J EAE mice showed evidence of an increase in oxidized nucleosides in layers $2 / 3$ of the subpial cortex, most prominently in the nucleolus (likely visualizing oxidized nuclear DNA) and in the cytoplasm (likely visualizing oxidized RNA) and to a lesser extent in the nuclear envelope of neurons (visualized in Figure 8, I-K, and quantified in Figure 8L). Moreover, treatment with BAF312 resulted in a nonsignificant reduction (1.3-fold) in $8 \mathrm{OHG}^{+}$cell count (Figure $8 \mathrm{~L}$ ). In summary, adoptive transfer of Th17 cells into SJL/J recipient mice 
resulted in demyelination, microgliosis, erosion of the glial limitans, and enhanced metabolic oxidative injury in the subpial cortex, whereas inhibition of $\mathrm{S}_{1} \mathrm{P}_{1,5}$ signaling partially reduced these pathological features.

\section{Discussion}

The goals of the current study were to ascertain the impact of meningeal TLT formation on pathology in the underlying cortex in $\mathrm{A} / \mathrm{T} \mathrm{SJL} / \mathrm{J}$ EAE and to determine the impact of timed modulation of leukocyte entry into the brain on immunological and pathological correlates of clinical disease. We found that adoptive transfer of PLP-primed T cells resulted in the formation of meningeal TLTs that are associated with a gradient of demyelination and microglia/macrophage accumulation as well as the degradation of the glial limitans and oxidative injury in the subpial cortex, reproducing some aspects of the pathology seen in MS patients with acute (18) and progressive disease (5). Treatment with BAF312 5 days after the adoptive transfer of Th17 cells arrested the propagation of these pathological features, suggesting that $\mathrm{S}_{\mathrm{PR}} \mathrm{R}_{1,5}$ signaling is required to maintain meningeal TLTs and cortical pathology.

Traditional rodent EAE models generally show little or no pathology in the cerebral cortex (20), particularly in the cortical gray matter. An exception is a model whereby rats are immunized with myelin (MOG) and supplemented with injections of proinflammatory cytokines (e.g., TNF- $\alpha$ or IFN- $\gamma$ ) into the brain parenchyma $(21,27)$ or into the subarachnoid space of the brain meninges $(20)$. In these models, cortical demyelination is accompanied by complement (C5b-9) deposition and high titers of anti-MOG antibodies $(20,27)$. In a separate study examining mice receiving stereotaxic injections of TNF- $\alpha$ or IFN- $\gamma$, only mice that express a B cell receptor transgene specific for MOG (based on the demyelinating 8-18C5 antibody) develop subpial cortical pathology (21). These data suggest that anti-MOG antibodies are a necessary requisite for cortical injury in the context of local administration of TNF- $\alpha$ or IFN- $\gamma$. However, recent evidence implicates antibody-independent mechanisms in the generation of cortical lesions, in particular oxidative injury (3), calling into question whether anti-MOG antibodies generated in some EAE models are relevant to the pathological processes that occur within subpial cortical lesions in patients with MS (34). To determine whether anti-myelin antibodies were associated with cortical injury in our model, we chose to examine anti-MOG antibody responses because anti-MOG antibodies have been shown to contribute to brain pathology in the context of EAE (35) and because MOG is the outermost myelin-associated protein (therefore a reasonable target for antibodies) as well as the most dominant antibody response during EAE (36). Conveniently, the availability of serum from mice in which EAE was induced by immunization with hMOG served as a technical positive control for comparison. However, when comparing with positive control serum, we did not find evidence of anti-MOG antibody generation in the serum of A/T SJL/J EAE mice. Although it remains possible that other anti-myelin antibody responses against less accessible antigens (i.e., PLP) may have been induced by adoptive transfer of Th17 cells, we view this as unlikely because an anti-myelin antibody response would entail liberation of myelin antigens into draining lymph nodes followed by a de novo germinal center response, and this would need to occur during the brief period following adoptive transfer of Th17 cells. In summary, despite the absence of anti-MOG antibodies, we observed considerable damage to areas of the cortex that lie adjacent to meningeal TLT, implying that in this model, anti-MOG antibodies are not necessary to induce cortical demyelination.

The lack of detectable anti-MOG antibodies in our model is of clinical relevance. Specifically, some diseases that originally were regarded as part of the heterogeneous spectrum of MS now are identified as distinct entities, which can be differentiated from classical MS by the presence of specific autoantibodies (37). For example, although MOG antibody-associated disease presents with a similar pathology to MS, it is a different disease than MS. Importantly, the mechanisms that induce pathology in MOG antibody-associated disease and in MS are distinct. Although demyelination and neurodegeneration in MOG antibody-associated disease are driven by anti-MOG autoantibodies, in MS subpial cortical demyelination is thought to be driven by soluble molecules that are produced by immune cells residing in the leptomeninges. These molecules can then diffuse across a disrupted glial limitans into the brain parenchyma, causing subpial injury. Thus, it is imperative to establish mouse models that show evidence of subpial pathology independent of anti-MOG antibodies to study what other factors may mediate cortical demyelination and injury.

Recent findings support the concept that such soluble factors can be produced by $\mathrm{T}$ cells $(5,38,39)$. In the $\mathrm{A} / \mathrm{T} \mathrm{SJL} / \mathrm{J}$ EAE model system in SJL/J mice, we previously showed that a reduction in meningeal stromal cell remodeling could be achieved by administering anti-IL-17A/IL-17F/IL-22 blocking antibodies by the intracerebral ventricular route, and this corresponded to a diminution in EAE symptoms (22). 
Extending these findings, we find that, in terms of T cells, BAF312 treatment reduced the accumulation of endogenous $\mathrm{CD}^{+} \mathrm{T}$ cells when expressed as a frequency of total live cells in the brain. We also found that BAF312 treatment had a preferential effect on the capacity of transferred CD4 ${ }^{+} \mathrm{T}$ cells - as opposed to endogenous $\mathrm{T}$ cells - to produce Th17 cytokines when expressed as a frequency of $\mathrm{CD}^{+} \mathrm{T}$ cells. These data imply that BAF312 treatment operates on endogenous $\mathrm{T}$ cells by preventing their accumulation in the CNS, whereas it operates on transferred (preactivated) $\mathrm{T}$ cells by changing their cytokine profile (reducing Th17 cytokine production). The impact of BAF312 on the accumulation of endogenous CD4 ${ }^{+}$but not transferred $\mathrm{CD}^{+} \mathrm{T}$ cells makes sense given that transferred $\mathrm{T}$ cells are introduced into the circulation and quickly migrate to the CNS (22), potentially precluding them from the lymph node retentive effects of BAF312. In contrast de novo recruitment of endogenous $\mathrm{CD}^{+} \mathrm{T}$ cells to the CNS from lymph node tissues would be regulated by S1P and would thus be affected by BAF312. In terms of why Th17 cytokines were uniquely affected in the transferred (preactivated/polarized) $\mathrm{CD} 4^{+} \mathrm{T}$ cell pool, this also makes sense because transferred $\mathrm{T}$ cells (which are preactivated in vitro) exhibit increased capacity for proinflammatory cytokine production compared with endogenous $\mathrm{T}$ cells (see Figure 5, C vs. E), possibly rendering these $\mathrm{T}$ cells more responsive to BAF312 treatment compared with their endogenous counterparts. Indeed, Th17 cells have been shown to be directly affected by S1P signaling (40), and FTY720 treatment (which prevents lymphocytes from responding to S1P) results in a suppressed Th17 response via downregulation of STAT3 phosphorylation (41). Interestingly, modulation of $\mathrm{S}_{1} \mathrm{P}_{1,5}$ signaling with BAF312 treatment at day 5 , but not day 8 , after adoptive transfer attenuates cortical pathology. At day 5, adoptively transferred $\mathrm{T}$ cells have already migrated into the brain (22). Thus, this suggests that a wave of "pioneer" $\mathrm{T}$ cells entering the brain between day 5 and day 8 sets the stage for cortical injury and clinical disease.

Notably, we also found that BAF312 treatment reduced the density of B220+ cells in the meninges. It is possible that loss of meningeal B cell infiltration contributes to the beneficial effects of BAF312 treatment. However, it is difficult to ascribe a direct mechanistic role for the observed reduction in meningeal B cells in the efficacy of BAF312 treatment, because the absence of B cells can change the balance of cytokines (such as B cell activating factor) that can favor the accumulation of regulatory B cell subsets with antiinflammatory properties (42). In the future it will be of interest to test the impact of different B cell subsets, including plasma cells, on the formation/maintenance of TLT and the development of subpial pathology in $\mathrm{A} / \mathrm{T}$ SJL EAE.

Oxidative injury and mitochondrial damage, which are key components of MS pathology $(3,31,32,43)$, are only partially reflected in current EAE models (33) but reproduced to some extent in the A/T SJL/J EAE mice, as shown by increased mtHsp70 and oxidized DNA/RNA, particularly in neurons. The observation that signs of oxidative injury and mitochondrial damage occur in parallel with a marked increase in the density of microglia/macrophages supports a process of microglial oxidative burst in the cortex that has been proposed as a mechanism of neuroaxonal injury in chronic MS (44). Thus, the A/T SJL/J model allows one to study acute cortical injury in a mouse setting that shares several features with the cortical pathology observed in the human MS brain. However, although the A/T SJL/J EAE model reproduces important aspects of MS cortical pathology and meningeal inflammation $(3,18,31,32,43)$, it has limitations because it does not capture all aspects of the disease $(5,45)$. For example, in MS, the inflammatory reaction in the brain is dominated by $\mathrm{CD}^{+} \mathrm{T}$ lymphocytes (45). However, like other models of EAE (46), our model is driven by CD4 ${ }^{+} \mathrm{T}$ cells. In addition, MS gray matter lesions show a gradient of neuronal loss that is more pronounced in layers proximal to the meninges (5). However, our NeuN staining indicates that this feature is not recapitulated in the A/T SJL/J EAE model. We hypothesize that other variables contribute to neuronal loss in the MS cortex, including a protracted period of chronic inflammation that may last for decades. In line with this, it would be interesting to determine whether factors such as age and environmental cues (e.g., the microbiome) may synergize with Th17 cells to affect additional features of cortical pathology. Nevertheless, the A/T SJL/J EAE model is, to our knowledge, the only model that mimics subpial cortical demyelination that is associated with aggregates of meningeal immune cells and is not dependent on anti-MOG antibodies. As such, it serves as a useful tool to test the translational value of disease-modifying therapies in MS.

Indeed, in the current study, we used this model to test the efficacy and potential mechanisms of action of BAF312. Although a subpopulation of secondary-progressive MS patients with more active disease exhibited a clinical response to BAF312 (47), how BAF312 exerts its protective effect is still unclear. Understanding its mode of action is extremely important to guide and improve new therapies. On one hand, BAF312 is thought to have antiinflammatory properties by blocking the egress of lymphocytes from the 
secondary lymphoid organs, thereby preventing their entry into the CNS (48). On the other hand, it has been shown that BAF312 readily crosses the blood-brain barrier and therefore potentially exerts beneficial effects by a interaction with brain cells, such as astrocytes (49), microglia (49), oligodendrocytes (50), or neurons (51). Using our model, we now show a third possible mode of action of BAF312 — specifically that BAF312 reduces the ability of "pioneer" $\mathrm{T}$ cells to produce Th17 cytokines in the brain and prevents the accumulation of $\mathrm{T}$ and $\mathrm{B}$ cells in the meningeal TLT, corresponding with a reduction in demyelination, microglia/ macrophage accumulation, and oxidative injury, as well as a preservation of the glial limitans in the cortex. The development of ultra-high field (such as 7-tesla) MRI, as a promising tool to visualize leptomeningeal enhancement (52), could aid in the stratification of patients who may benefit from BAF312 therapy.

\section{Methods}

Animals. Female 6- to 10-week-old SJL/J CD45.1 ${ }^{+}$mice were obtained from Envigo. Female 6- to 10-weekold SJL/J CD45.2 $2^{+}$mice were derived from C57BL/6 mice via speed congenics and backcrossed onto $\mathrm{SJL} / \mathrm{J}$ mice (Envigo) at the University of Toronto animal facilities. Aicda ${ }^{-/-}$mice were obtained from Tasuku Honjo, Kyoto University (53). For the ELISA analysis, 5- to 7-week-old C57BL/6 mice were sourced from The Jackson Laboratory. Animals were housed at the University of Toronto animal facilities under specific pathogen-free conditions, in a closed caging system with a 12-hour light/12-hour dark cycle. They were provided with a standard irradiated chow diet (Teklad; Envigo, 2918) and acidified water (reverse osmosis and ultraviolet sterilized) ad libitum.

$A / T E A E$ and active EAE. A/T SJL/J EAE was performed as described before (22). Briefly, CD45.2 ${ }^{+}$ donor mice were immunized with $100 \mu \mathrm{gLP}_{139-151}$ (HSLGKWLGHPDKF; Canpeptide) in an emulsion of incomplete Freund's adjuvant (BD Difco), supplemented with $200 \mu \mathrm{g}$ Mycobacterium tuberculosis H37 Ra (BD Difco, 231141) in a total volume of $300 \mu \mathrm{L}$ administered as three $100 \mu \mathrm{L}$ subcutaneous injections on the back and flanks. On day 9 after immunization, cells from the spleen and draining lymph nodes (inguinal, cervical, axillary, and brachial) were harvested. In some, but not all experiments (as indicated in the figure legends), $\mathrm{CD}^{+}$cells were selected from the spleen and LNs using a mouse CD4 ${ }^{+} \mathrm{T}$ Cell Isolation Kit (Miltenyi Biotec). CD4 ${ }^{+} \mathrm{T}$ cells were then restimulated ex vivo with $\operatorname{PLP}_{139-151}(10 \mu \mathrm{g} / \mathrm{mL})$ in the presence of anti-IFN- $\gamma$ (20 $\mu \mathrm{g} / \mathrm{mL}$, Bioceros), anti-IL-4 (20 $\mu \mathrm{g} / \mathrm{mL}$, Bioceros), and IL-23 (10 ng/mL, R\&D Systems) for 72 hours at $37^{\circ} \mathrm{C}$. In total, $2 \times 10^{6} \mathrm{CD}^{+} \mathrm{T}$ cells were injected intraperitoneally into SJL/J CD $45.1^{+}$recipient mice.

EAE induced by immunization with $\mathrm{hMOG}$ was used to compare anti-MOG antibody responses with A/T SJL/J EAE. Briefly, C57BL/6 or $\mathrm{Aicda}^{-/-}$littermate control mice were immunized with recombinant hMOG - corresponding to the extracellular domain of human MOG - provided by C. Linington (University of Glasgow, Glasgow, United Kingdom) and N. Ruddle (Yale School of Medicine, New Haven, Connecticut, USA) and prepared as previously described (28). Mice 6-8 weeks of age were immunized subcutaneously with $100 \mu \mathrm{g}$ of hMOG emulsified 1:1 in incomplete Freund's adjuvant containing $4 \mathrm{mg} /$ $\mathrm{mL}$ Mycobacterium tuberculosis (H37 Ra). Mice also received two 400-ng i.p. doses of pertussis toxin (List Biological Laboratories) on the day of immunization and 48 hours later.

Clinical assessment of EAE. For clinical assessment of EAE disease, recipient mice were weighed daily and scored according to a composite scale that we $(28)$ and others $(54,55)$ have previously published. Briefly, the composite scale measures mobility impairments in each limb and the tail. Each limb is graded from 0 (asymptomatic) to 3 (complete paralysis), and the tail is graded from 0 (asymptomatic) to 2 (limp tail). Assessment of the righting reflex is scored from 0 to 2, with 0 being assigned for a normal righting reflex, 1 for slow righting reflex, and 2 for a delay of more than $5 \mathrm{~s}$ in the righting reflex. Each criterion was measured in 0.5 increments. Thus, the composite score ranges from 0 (nonsymptomatic) to 16 (fully quadriplegic mouse with limp tail and significantly delayed righting reflex). The clinical assessment of EAE disease was conducted blindly and by 2 investigators, independently.

Animal nursing. During EAE, we increased daily nursing to improve survival by placing cages on heat pads $\left(37^{\circ} \mathrm{C}\right)$ with Pure-o'Cel paperless bedding. Diet was supplemented with breeder mash (Envigo Teklad, 2919) to help mice gain or maintain body weight, and subcutaneous injections of 1-1.5 mL of lactated Ringer's solution, as well as 5\% dextrose for caloric supplementation, were provided 1-3 times daily depending on symptoms and signs of dehydration.

BAF312 (siponimod) treatment. BAF312 (siponimod) was synthesized by Novartis. BAF312 was administered in SJL/J CD45.1 $1^{+}$recipient mice by daily oral gavage at a dose of $3 \mathrm{mg} / \mathrm{kg}$, diluted in CMC/Na medium viscosity (MilliporeSigma) in an application volume of $10 \mathrm{~mL} / \mathrm{kg}$, starting at 3, 5, or 8 days after 
adoptive transfer. Control mice received daily oral gavage treatment with $\mathrm{CMC}$ (vol/vol) at the same time points after adoptive transfer. Mice were randomized into either the treatment or control group.

Tissue harvest. Animals were analyzed at the peak phase of disease, 11 days after adoptive transfer. At the time of sacrifice, blood was collected via cardiac puncture and transferred into lithium heparin-coated microcapillary tubes (BD). Mice were intracardially perfused with $30 \mathrm{~mL}$ of ice-cold PBS. Brains were dissected from the skull and spinal columns. The 2 brain hemispheres were sagittally divided. One hemisphere was used for the generation of a single-cell suspension and subsequent analysis by flow cytometry, whereas the other hemisphere was either postfixed in 10\% buffered formalin (MilliporeSigma) for 1 week prior to being processed into paraffin for pathological analysis or postfixed in $10 \%$ buffered formalin for 1 hour followed by immersion in $30 \%$ sucrose overnight at $4^{\circ} \mathrm{C}$, then placed in plastic molds with optimal cutting temperature mounting medium (Thermo Fisher Scientific), frozen in a bath of 2-O-methylbutane on dry ice (Fisher Chemical, Thermo Fisher Scientific), and stored at $-80^{\circ} \mathrm{C}$ until they were sectioned.

Preparation and flow cytometry of brain single-cell suspensions. Preparation of brain single-cell suspensions was performed as described before (22). Briefly, brain hemispheres were mashed in digestion buffer (10 $\mathrm{mM}$ HEPES, $150 \mathrm{mM} \mathrm{NaCl}, 1 \mathrm{mM} \mathrm{MgCl}, 5 \mathrm{mM} \mathrm{KCl}$, and $1.8 \mathrm{mM} \mathrm{CaCl}_{2}$ in $\mathrm{HBSS}_{2}$ buffer). To dissociate cells from their resident tissues, collagenase $\mathrm{D}$ (Roche) was added to a final concentration of $1 \mathrm{mg} / \mathrm{mL}$ and DNAseI (Roche) to a final concentration of $60 \mu \mathrm{g} / \mathrm{mL}$ to each sample. Brain samples were incubated at $37^{\circ} \mathrm{C}$ for 30 minutes, mixed with a pipette tip, and reincubated for an additional 15 minutes. Upon removal, a final concentration of $1 \mathrm{mM}$ EDTA $\mathrm{pH} 8.0$ was added to each sample and incubated at room temperature for 10 minutes. Samples were then filtered through a $70-\mu \mathrm{m}$ filter and washed twice with ice-cold PBS. Cells were resuspended into a 30\% Percoll (GE Healthcare) solution and centrifuged to separate the fat from the cells. Collected lymphocytes were washed twice in ice-cold PBS and resuspended in complete RPMI (10\% FBS from Gibco, Thermo Fisher Scientific; L-glutamine from MilliporeSigma, sodium pyruvate from MilliporeSigma, penicillin from MilliporeSigma, streptomycin from MilliporeSigma, HEPES pH 7.0 from Gibco, Thermo Fisher Scientific; and $\beta$-mercaptoethanol from Gibco, Thermo Fisher Scientific, in RPMI1640 medium from MilliporeSigma). Cells were counted with a hemocytometer and plated at a density of 300,000 cells/well. Following incubation at $37^{\circ} \mathrm{C}$ for 5 hours with an intracellular cytokine restimulation buffer (PMA [MilliporeSigma, stock $500 \mu \mathrm{g} / \mathrm{mL}$ ] used at 1:100,000; ionomycin [MilliporeSigma, stock 0.5 $\mathrm{mg} / \mathrm{mL}$ ] used at 1:1000; and Brefeldin A [eBioscience, Thermo Fisher Scientific, stock 100x] used at 1:1000 in complete RPMI), cells were collected, washed twice in ice-cold PBS, and stained for flow cytometry.

Brain lymphocytes were then stained with LIVE/DEAD Fixable Aqua (Life Technologies, Thermo Fisher Scientific) at 1:1000 in PBS for 30 minutes at $4^{\circ} \mathrm{C}$. This was immediately followed by staining with anti-CD4-FITC (eBioscience, Thermo Fisher Scientific), anti-CD8-BV711 (eBioscience, Thermo Fisher Scientific), anti-CD3-APC (eBioscience, Thermo Fisher Scientific), anti-B220-BV605 (BioLegend), anti-CD19-PE (eBioscience, Thermo Fisher Scientific), anti-CD45.1-APCeF780 (eBioscience, Thermo Fisher Scientific), and anti-CD45.2-eF450 (eBioscience, Thermo Fisher Scientific) in PBS with 2\% FBS and $1 \mathrm{mg} / \mathrm{mL}$ Fc block. Cells were then permeabilized with CytoFix/CytoPerm (BD Biosciences) for 20 minutes at $4^{\circ} \mathrm{C}$. After washing twice with $1 \times$ Perm/Wash buffer (BD Biosciences), intracellular markers were stained with anti-GM-CSF-FITC (eBioscience, Thermo Fisher Scientific), anti-IL-17A-PerCP-Cy5.5 (eBioscience, Thermo Fisher Scientific), and anti-IFN- $\gamma$-PE-Cy7 (eBioscience, Thermo Fisher Scientific) in $1 \times$ Perm/Wash buffer with $1 \mathrm{mg} / \mathrm{mL}$ Fc block. Reagents are listed in Supplemental Table 2. Cells were acquired by FACS using the LSRFortessa X-20 II instrument (BD Biosciences) and FACSDiva software (BD Biosciences). Data analysis was done with FlowJo V10.5.3 software.

ELISA. Serum samples were obtained at the chronic phase of the disease for C57BL/6 (positive control for anti-MOG IgG) or $\mathrm{Aicda}^{-/-}$mice (positive control for anti-MOG IgM and negative control for anti-MOG IgG) or at day 11 after adoptive transfer for SJL/J recipient mice. Blood was collected and centrifuged at 1500 $g$ for 10 minutes to obtain serum. ELISAs were performed as described before (28). Briefly, serum was diluted to a starting dilution of 1:20, 1:50, or 1:500 and then serially diluted 1:2. Anti-MOG antibodies were assessed using Sensolyte Anti-Mouse MOG $_{1-125}$ IgG Quantitative ELISA Kit (AnaSpec, AS-55156) according to manufacturer instructions or modified as described below. Briefly, 96-well plates coated with mouse $\mathrm{MOG}_{1-125}$ were incubated with sera samples at the appropriate dilutions for 1 hour at room temperature with gentle shaking. Following incubation, wells were washed 5 times with wash buffer, and goat anti-mouse IgG-HRP secondary antibody was added to each well for 1 hour at room temperature. After 5 washes, bound mouse MOG-specific antibodies were detected with 3,3',5,5'-Tetramethylbenzidine, and optical density at $450 \mathrm{~nm}$ 
was measured using a spectrophotometer. For the detection of anti-MOG IgM, the goat anti-mouse IgG-HRP was substituted for anti-IgM-HRP at 1:5000 dilution (BD). In anti-MOG IgM assays, dilution curves were generated using a 4-parameter logistic regression for each sample, and the $\mathrm{IC}_{50}$ of each sample was normalized against a pooled positive control that was run on every plate. The positive control was made up of pooled sera from 3 C57BL/ 6 mice immunized with hMOG and sacrificed at the chronic phase of disease.

Histology and immunohistochemistry. Seven-micron paraffin sagittal sections of mouse brain were collected from the midline, mounted on Superfrost Plus glass slides (Knittel Glass), and dried in the oven (Precision compact oven, Thermo Fisher Scientific) overnight at $37^{\circ} \mathrm{C}$. Paraffin sections were deparaffinated in xylene (Fisher Chemical, Thermo Fisher Scientific) and rehydrated through a series of ethanol washes. Histology was performed using standard HE stain and then placed in xylene before being coverslipped with Entellan mounting media (MilliporeSigma).

For immunohistochemistry, deparaffinized and rehydrated brain sections were incubated in $0.3 \% \mathrm{H}_{2} \mathrm{O}_{2}$ in methanol for 20 minutes to block endogenous peroxidase activity. Epitopes were exposed by heat-induced antigen retrieval in $10 \mathrm{mM}$ sodium citrate buffer $(\mathrm{pH}$ 6.0) or $10 \mathrm{mM}$ Tris $1 \mathrm{mM}$ EDTA ( $\mathrm{pH}$ 9.0), depending on the antibody used (see Supplemental Table 2) in a pressure cooker placed inside a microwave set at high power ( $~ 800$ watts) for 15 minutes. The nonspecific binding of antibodies was blocked using $10 \%$ normal goat serum (DAKO) in PBS for 20 minutes at room temperature.

Primary antibodies to detect myelin (PLP), oligodendrocytes (Tppp/p25), microglia/macrophages (Iba-1), astrocytes (GFAP), neurons (NeuN), mitochondrial metabolic stress ( $\mathrm{mtHsp} 70)$, and oxidative injury (8OHG) were diluted in Normal Antibody Diluent (Immunologic) according to the dilutions shown in Supplemental Table 2. Sections were incubated in the primary antibody solution overnight at $4^{\circ} \mathrm{C}$. The following day, sections were incubated with the Post Antibody Blocking Solution for monoclonal antibodies (Immunologic) diluted 1:1 in PBS for 15 minutes at room temperature (RT). Detection was performed by incubating the sections in the secondary poly-HRP-goat anti-mouse/rabbit/rat IgG (Immunologic) antibodies diluted 1:1 in PBS for 30 minutes at RT followed by incubation in DAB (Vector Laboratories) as the chromogen. Counterstaining was performed with hematoxylin (MilliporeSigma) for 10 minutes. The sections were subsequently dehydrated through a series of ethyl alcohol solutions and then placed in xylene before being coverslipped with Entellan mounting medium. Sections stained with secondary antibody alone were included as negative controls. For the $8 \mathrm{OHG}$ staining, the specificity of the anti-8OHG antibody was confirmed by (a) comparison with adjacent sections in which the primary antibody was omitted to assess whether the secondary antibody alone contributed to the $8 \mathrm{OHG}$ staining signal or (b) pretreatment with $100 \mu \mathrm{g} / \mathrm{mL}$ of RNAse A (BioBasic, RB0473) in TEN buffer (10 mM Tris- $\mathrm{HCl} \mathrm{pH} 7.4,1 \mathrm{mM}$ EDTA, $400 \mathrm{mM} \mathrm{NaCl}$ ) for 1 hour at $37^{\circ} \mathrm{C}$ to assess whether the (oxidized) RNA in the tissue was the source of the $8 \mathrm{OHG}$ signal or (c) omission of the $\mathrm{H}_{2} \mathrm{O}_{2}$ step to assess whether $\mathrm{H}_{2} \mathrm{O}_{2}$-mediated oxidation during processing contributed to the $8 \mathrm{OHG}$ staining signal. Control stainings (a) and (b) were negative. Control staining (c) did not alter the staining pattern obtained by including the $\mathrm{H}_{2} \mathrm{O}_{2}$ step in the protocol. Altogether, the control staining showed the specificity of the anti-8OHG antibody.

Immunofluorescence. Preparation of frozen tissues for immunofluorescence staining was performed as described before (22). Briefly, 7- $\mu \mathrm{m}$ frozen sagittal sections of mouse brain were collected from the midline, mounted on Superfrost Plus glass slides (Knittel Glass), and postfixed in acetone for 10 minutes at $-20^{\circ} \mathrm{C}$, before being stored at $-80^{\circ} \mathrm{C}$ until they were stained. On the day of staining, the slides were brought at room temperature and incubated in ice-cold $70 \%$ ethanol for 10 minutes, to reduce the autofluorescence signal derived from the fatty myelin sheets. Slides were subsequently incubated in blocking buffer consisting of $10 \%$ normal goat serum (DAKO) in 5\% bovine serum albumin in PBS, supplemented with Fc block (1:100) to block nonspecific binding sites. Sections were then incubated overnight at $4^{\circ} \mathrm{C}$ with a cocktail of primary antibodies, diluted in blocking buffer, to detect either fibronectin and CD45 ${ }^{+}$cells or $\mathrm{CD}^{+}$and $\mathrm{B} 220^{+}$cells (see Supplemental Table 2). Primary antibodies were either directly conjugated or were detected using fluorochrome-conjugated secondary antibodies (MilliporeSigma) diluted in PBS. Sections were washed in PBS, air dried, and mounted in aqueous mounting medium (R\&D Systems). The immunofluorescent staining was visualized under the microscope (Axio Imager Z1, Zeiss), using the appropriate filters, and connected to a digital camera (AxioCam 506 mono, Zeiss) and the Zen pro 2.0 imaging software (Zeiss). Each staining was performed in 2 sets of nonconsecutive brain sections, which were used for quantification.

Quantitative analysis of immunostaining. For each marker analyzed, 2-3 stained sections were scored for each mouse. To ensure that similar areas were compared across mice, sections were collected from the midline outward in each brain hemisphere. Sagittal sections were collected to ensure that many 
anatomical regions of the brain (from the olfactory bulbs to the brainstem) were captured at once. We screened entire brain sections under the microscope, but for the analysis we focused on areas where TLTs were usually observed at acute disease stage (meninges outlining the midbrain between the superior and the inferior colliculus and the somatosensory cortex). For some but not all mice, brain sections were randomly sampled across the brain hemisphere. However, because we did not stain/analyze the entire brain, the possibility remains that we missed information, such as the presence of TLT, in areas of the brains that were not sectioned/analyzed. Red/green/blue (RGB) images of HE, PLP, Tppp/p25, Iba-1, GFAP, $\mathrm{NeuN}, \mathrm{mtHsp} 70$, and $8 \mathrm{OHdG}$ stains from brain sections were acquired at original magnification $\times 20$ using a light microscope (Axioscope, Zeiss), connected to a digital camera (AxioCam MRc, Zeiss), and Zen pro 2.0 imaging software (Zeiss). ImageJ $1.15 \mathrm{~s}$ (National Institutes of Health) was used to measure the extent of demyelination in the subpial cortex and the extent of mitochondrial metabolic stress and to measure the surface area analyzed in the digital images.

The quantification of subpial demyelination and mitochondrial metabolic stress was performed on PLP- and mtHsp70-immunostained sections, respectively, on equivalent regions of interest (ROIs). The RGB images were separated into single-color channels using the color deconvolution plug-in in ImageJ. The single-color channel for PLP or mtHsp70 was subjected to thresholding to create a mask that captured the specific staining. The area fraction measurement was applied to each ROI to quantify the percentage of thresholded staining (see Supplemental Figure 5). The scale represents the percentage of $\mathrm{PLP}^{+}$or $\mathrm{mtHsp} 70^{+}$cortical area in each brain section examined.

The densities of Tppp/p25+ $\mathrm{Iba}^{+}, \mathrm{GFAP}^{+}, \mathrm{NeuN}^{+}$, and $8 \mathrm{OHG}^{+}$cells were determined using a morphometric grid (at original magnification $\times 200$ ), and the data were expressed as cells per $\mathrm{mm}^{2}$.

For the histological assessment of TLTs, a quantification of TLTs' size and density (cells $/ \mathrm{mm}^{2}$ of TLT) of $\mathrm{B}_{2} 20^{+} \mathrm{B}$ cells and $\mathrm{CD}^{+} \mathrm{T}$ cells was performed. For all quantifications of staining, the experimental groups were blinded to the investigators. The ROIs were selected either at cortical layers 1 and $2 / 3$ or at the midbrain (as indicated in the text).

Statistics. Statistical analyses were performed with GraphPad Prism (GraphPad Software). For analysis of the EAE clinical disease course and analysis of the gradient of histological staining, 2-way ANOVA was used. For analysis of all other histology, the variability of distribution was assessed by Shapiro-Wilk normality test. For non-normally distributed data, the nonparametric Mann-Whitney $U$ (between 2 groups) or Kruskal-Wallis (between multiple groups) test was performed. For analysis of the FACS and ELISA data, Mann-Whitney $U$ was used. Statistically significant $P$ values $(P<0.05)$ are indicated in the figure legends.

Study approval. All animal experiments were conducted in accordance with institutional guidelines, with ethical approval from the University of Toronto Faculty of Medicine Animal Care Committee.

\section{Author contributions}

LAW and DSWL carried out the A/T SJL/J EAE induction protocol. LAW maintained the SJL/J CD45.2 mouse colony and performed the flow cytometry experiments. DSWL analyzed the flow cytometry data and carried out the ELISA experiments. AS and XIM monitored the EAE mice, performed the immunostaining, and participated in the data analysis. AW and IN assisted with the collection and processing of the mouse tissue. NP and BNH advised on the experiments. VR monitored the EAE mice, assisted in the collection and processing of the mouse tissue, supervised the immunostaining experiments, and participated in the data analysis. JLG designed and supervised the study. VR and JLG wrote the manuscript. Method used in assigning the shared authorship: LAW initiated and carried out the adoptive transfer studies, and DSWL performed and analyzed all the flow cytometry and ELISAs.

\section{Acknowledgments}

We thank Novartis for providing the BAF312 (siponimod) for the inhibition studies. This work was funded by a team grant from the MS Society Research Foundation to JLG, a sponsored research agreement with Novartis to JLG, and the National Multiple Sclerosis Society Research Grant RR-1602-07777 to VR.

Address correspondence to: Valeria Ramaglia, University of Toronto, Medical Sciences Building, Department of Immunology, 1 King's College Circle, Room 7310, Toronto, Ontario, M5S 1A8, Canada. Phone: 416.978.4119; Email: v.ramaglia@utoronto.ca. 
1. Bø L, Vedeler CA, Nyland HI, Trapp BD, Mørk SJ. Subpial demyelination in the cerebral cortex of multiple sclerosis patients. J Neuropathol Exp Neurol. 2003;62(7):723-732.

2. Kuhlmann T, Ludwin S, Prat A, Antel J, Brück W, Lassmann H. An updated histological classification system for multiple sclerosis lesions. Acta Neuropathol. 2017;133(1):13-24.

3. Fischer MT, et al. Disease-specific molecular events in cortical multiple sclerosis lesions. Brain. 2013;136(Pt 6):1799-1815.

4. Lucchinetti CF, et al. Inflammatory cortical demyelination in early multiple sclerosis. N Engl J Med. 2011;365(23):2188-2197.

5. Magliozzi R, et al. A Gradient of neuronal loss and meningeal inflammation in multiple sclerosis. Ann Neurol. 2010;68(4):477-493.

6. Magliozzi R, et al. Meningeal B-cell follicles in secondary progressive multiple sclerosis associate with early onset of disease and severe cortical pathology. Brain. 2007;130(Pt 4):1089-1104.

7. Filippi M, et al. Intracortical lesions: relevance for new MRI diagnostic criteria for multiple sclerosis. Neurology. 2010;75(22):1988-1994.

8. Calabrese M, et al. Widespread cortical thinning characterizes patients with MS with mild cognitive impairment. Neurology. 2010;74(4):321-328.

9. Fisniku LK, et al. Gray matter atrophy is related to long-term disability in multiple sclerosis. Ann Neurol. 2008;64(3):247-254.

10. van Horssen J, Brink BP, de Vries HE, van der Valk P, Bø L. The blood-brain barrier in cortical multiple sclerosis lesions. J Neuropathol Exp Neurol. 2007;66(4):321-328.

11. Peterson JW, Bö L, Mörk S, Chang A, Trapp BD. Transected neurites, apoptotic neurons, and reduced inflammation in cortical multiple sclerosis lesions. Ann Neurol. 2001;50(3):389-400.

12. Brink BP, Veerhuis R, Breij EC, van der Valk P, Dijkstra CD, Bö L. The pathology of multiple sclerosis is location-dependent: no significant complement activation is detected in purely cortical lesions. J Neuropathol Exp Neurol. 2005;64(2):147-155.

13. Watkins LM, et al. Complement is activated in progressive multiple sclerosis cortical grey matter lesions. J Neuroinflammation. 2016;13(1):161.

14. Calabrese M, Magliozzi R, Ciccarelli O, Geurts JJ, Reynolds R, Martin R. Exploring the origins of grey matter damage in multiple sclerosis. Nat Rev Neurosci. 2015;16(3):147-158.

15. Choi SR, et al. Meningeal inflammation plays a role in the pathology of primary progressive multiple sclerosis. Brain. 2012;135(Pt 10):2925-2937.

16. Haider L, et al. The topograpy of demyelination and neurodegeneration in the multiple sclerosis brain. Brain. 2016;139(Pt 3):807-815.

17. Howell OW, et al. Meningeal inflammation is widespread and linked to cortical pathology in multiple sclerosis. Brain. 2011;134(Pt 9):2755-2771.

18. Bevan RJ, et al. Meningeal inflammation and cortical demyelination in acute multiple sclerosis. Ann Neurol. 2018;84(6):829-842.

19. Pikor NB, Prat A, Bar-Or A, Gommerman JL. Meningeal tertiary lymphoid tissues and multiple sclerosis: a gathering place for diverse types of immune cells during CNS autoimmunity. Front Immunol. 2015;6:657.

20. Gardner C, Magliozzi R, Durrenberger PF, Howell OW, Rundle J, Reynolds R. Cortical grey matter demyelination can be induced by elevated pro-inflammatory cytokines in the subarachnoid space of MOG-immunized rats. Brain. 2013;136(Pt 12):3596-3608.

21. Lagumersindez-Denis $\mathrm{N}$, et al. Differential contribution of immune effector mechanisms to cortical demyelination in multiple sclerosis. Acta Neuropathol. 2017;134(1):15-34.

22. Pikor NB, et al. Integration of Th17- and lymphotoxin-derived signals initiates meningeal-resident stromal cell remodeling to propagate neuroinflammation. Immunity. 2015;43(6):1160-1173.

23. Androdias G, Reynolds R, Chanal M, Ritleng C, Confavreux C, Nataf S. Meningeal T cells associate with diffuse axonal loss in multiple sclerosis spinal cords. Ann Neurol. 2010;68(4):465-476.

24. Howell OW, et al. Extensive grey matter pathology in the cerebellum in multiple sclerosis is linked to inflammation in the subarachnoid space. Neuropathol Appl Neurobiol. 2015;41(6):798-813.

25. Serafini B, Rosicarelli B, Magliozzi R, Stigliano E, Aloisi F. Detection of ectopic B-cell follicles with germinal centers in the meninges of patients with secondary progressive multiple sclerosis. Brain Pathol. 2004;14(2):164-174.

26. Skjoerringe T, Lundvig DM, Jensen PH, Moos T. P25alpha/Tubulin polymerization promoting protein expression by myelinating oligodendrocytes of the developing rat brain. J Neurochem. 2006;99(1):333-342.

27. Merkler D, Ernsting T, Kerschensteiner M, Brück W, Stadelmann C. A new focal EAE model of cortical demyelination: multiple sclerosis-like lesions with rapid resolution of inflammation and extensive remyelination. Brain. 2006;129(Pt 8):1972-1983.

28. Galicia G, et al. Isotype-switched autoantibodies are necessary to facilitate central nervous system autoimmune disease in Aicda/- and Ung-/- mice. J Immunol. 2018;201(4):1119-1130.

29. Galicia G, Boulianne B, Pikor N, Martin A, Gommerman JL. Secondary B cell receptor diversification is necessary for T cell mediated neuro-inflammation during experimental autoimmune encephalomyelitis. PLoS One. 2013;8(4):e61478.

30. Sun Y, et al. Critical role of activation induced cytidine deaminase in experimental autoimmune encephalomyelitis. Autoimmunity. 2013;46(2):157-167.

31. Lassmann H, van Horssen J, Mahad D. Progressive multiple sclerosis: pathology and pathogenesis. Nat Rev Neurol. 2012;8(11):647-656.

32. Haider L, et al. Oxidative damage in multiple sclerosis lesions. Brain. 2011;134(Pt 7):1914-1924.

33. Schuh C, et al. Oxidative tissue injury in multiple sclerosis is only partly reflected in experimental disease models. Acta Neuropathol. 2014;128(2):247-266.

34. Lassmann $\mathrm{H}$. The changing concepts in the neuropathology of acquired demyelinating central nervous system disorders. Curr Opin Neurol. 2019;32(3):313-319.

35. Berg CT, Khorooshi R, Asgari N, Owens T. Influence of type I IFN signaling on anti-MOG antibody-mediated demyelination. J Neuroinflammation. 2017;14(1):127.

36. Morris-Downes MM, et al. Pathological and regulatory effects of anti-myelin antibodies in experimental allergic encephalomyelitis in mice. J Neuroimmunol. 2002;125(1-2):114-124.

37. Reindl M, Waters P. Myelin oligodendrocyte glycoprotein antibodies in neurological disease. Nat Rev Neurol. 2019;15(2):89-102.

38. Fadda G, et al. A surface-in gradient of thalamic damage evolves in pediatric multiple sclerosis. Ann Neurol. 2019;85(3):340-351.

39. Magliozzi R, et al. Inflammatory intrathecal profiles and cortical damage in multiple sclerosis. Ann Neurol. 2018;83(4):739-755. 
40. Eken A, et al. S1P deletion differentially affects TH17 and regulatory T cells. Sci Rep. 2017;7(1):12905.

41. Tsai HC, Huang Y, Garris CS, Moreno MA, Griffin CW, Han MH. Effects of sphingosine-1-phosphate receptor 1 phosphorylation in response to FTY720 during neuroinflammation. JCI Insight. 2016;1(9):e86462.

42. Rojas OL, et al. Recirculating intestinal IgA-producing cells regulate neuroinflammation via IL-10. Cell. 2019;177(2):492-493.

43. Witte ME, et al. Enhanced number and activity of mitochondria in multiple sclerosis lesions. J Pathol. 2009;219(2):193-204.

44. Lassmann H, van Horssen J. Oxidative stress and its impact on neurons and glia in multiple sclerosis lesions. Biochim Biophys Acta. 2016;1862(3):506-510

45. Machado-Santos J, et al. The compartmentalized inflammatory response in the multiple sclerosis brain is composed of tissue-resident CD8+ T lymphocytes and B cells. Brain. 2018;141(7):2066-2082.

46. Simmons SB, Pierson ER, Lee SY, Goverman JM. Modeling the heterogeneity of multiple sclerosis in animals. Trends Immunol. 2013;34(8):410-422.

47. Kappos L, et al. Siponimod versus placebo in secondary progressive multiple sclerosis (EXPAND): a double-blind, randomised, phase 3 study. Lancet. 2018;391(10127):1263-1273.

48. Matloubian M, et al. Lymphocyte egress from thymus and peripheral lymphoid organs is dependent on S1P receptor 1. Nature. 2004;427(6972):355-360.

49. O'Sullivan C, Schubart A, Mir AK, Dev KK. The dual S1PR1/S1PR5 drug BAF312 (Siponimod) attenuates demyelination in organotypic slice cultures. J Neuroinflammation. 2016;13:31.

50. Jackson SJ, Giovannoni G, Baker D. Fingolimod modulates microglial activation to augment markers of remyelination. J Neuroinflammation. 2011;8:76.

51. Gentile A, et al. Siponimod (BAF312) prevents synaptic neurodegeneration in experimental multiple sclerosis. J Neuroinflammation. 2016;13(1):207.

52. Harrison DM, et al. Leptomeningeal Enhancement at 7T in multiple sclerosis: frequency, morphology, and relationship to cortical volume. J Neuroimaging. 2017;27(5):461-468.

53. Muramatsu M, Kinoshita K, Fagarasan S, Yamada S, Shinkai Y, Honjo T. Class switch recombination and hypermutation require activation-induced cytidine deaminase (AID), a potential RNA editing enzyme. Cell. 2000;102(5):553-563.

54. Giuliani F, Metz LM, Wilson T, Fan Y, Bar-Or A, Yong VW. Additive effect of the combination of glatiramer acetate and minocycline in a model of MS. J Neuroimmunol. 2005;158(1-2):213-221.

55. Torre S, et al. USP15 regulates type I interferon response and is required for pathogenesis of neuroinflammation. Nat Immunol. 2017;18(1):54-63. 